\title{
Predicted Position Error Triggers Catch-Up Saccades during Sustained Smooth Pursuit
}

\author{
DOmri Nachmani, ${ }^{1}$ Jonathan Coutinho, ${ }^{1}$ Aarlenne Z. Khan, ${ }^{1,2}$ P.Philippe Lefèvre, ${ }^{3}$ and Gunnar Blohm ${ }^{1}$
}

https://doi.org/10.1523/ENEURO.0196-18.2019

${ }^{1}$ Centre for Neuroscience Studies, Queen's University, Kingston, Ontario, Canada K7L 3N6, 2VISATTAC, École d'Optométrie, Université de Montréal, Montreal, Ontario, Canada H3T 1P1, and ${ }^{3}$ Université Catholique de Louvain, Ottignies-Louvain-la-Neuve, Belgium MJ98+V6

\begin{abstract}
For humans, visual tracking of moving stimuli often triggers catch-up saccades during smooth pursuit. The switch between these continuous and discrete eye movements is a trade-off between tolerating sustained position error (PE) when no saccade is triggered or a transient loss of vision during the saccade due to saccadic suppression. de Brouwer et al. (2002b) demonstrated that catch-up saccades were less likely to occur when the target re-crosses the fovea within $40-180 \mathrm{~ms}$. To date, there is no mechanistic explanation for how the trigger decision is made by the brain. Recently, we proposed a stochastic decision model for saccade triggering during visual tracking (Coutinho et al., 2018) that relies on a probabilistic estimate of predicted PE (PE predictions, we hypothesized that saccade trigger time length and variability will increase when pre-saccadic predicted errors are small or visual uncertainty is high (e.g., for blurred targets). Data collected from human participants performing a double step-ramp task showed that large pre-saccadic $P E_{\text {pred }}\left(>10^{\circ}\right)$ produced short saccade trigger times regardless of the level of uncertainty while saccade trigger times preceded by small $\mathrm{PE}_{\text {pred }}$ $\left(<10^{\circ}\right)$ significantly increased in length and variability, and more so for blurred targets. Our model also predicted increased signal-dependent noise (SDN) as retinal slip (RS) increases; in our data, this resulted in longer saccade trigger times and more smooth trials without saccades. In summary, our data supports our hypothesized predicted error-based decision process for coordinating saccades during smooth pursuit.
\end{abstract}

Key words: catch-up; eye movements; pursuit; saccades; tracking; trigger

\section{Significance Statement}

The mechanism by which the brain decides when to trigger discrete catch-up saccades during continuous smooth pursuit has eluded researchers for decades. In this study, we present behavioral data complemented by computational model predictions in support of an intuitive trigger mechanism that relies on probabilistic estimation of future position error (PE). Our results add support for a common and shared sensorimotor process for saccades and pursuit. Furthermore, by linking motor control to statistical decision-making, we offer a novel perspective on how sensorimotor prediction and uncertainty modulate oculomotor tracking behavior.

\section{Introduction}

For humans to see an object clearly, its image must fall on a highly specialized region in the retina called the fovea. Foveal tracking of moving objects in the environ-

Received July 23, 2019; accepted November 28, 2019; First published December 20, 2019.

The authors declare no competing financial interests. ment is accomplished by smooth pursuit eye movements. However, due to visual feedback delay and sensorimotor noise, the eye can progressively lag behind the target

Author contributions: J.C. developed the computational saccade trigger model. O.N., A.Z.K., P.L., and G.B. contributed to the development of the experiment and analysis plan. O.N. coded the experiment and analysis pipeline, collected subject data, analyzed results, produced figures, and wrote the manuscript. 


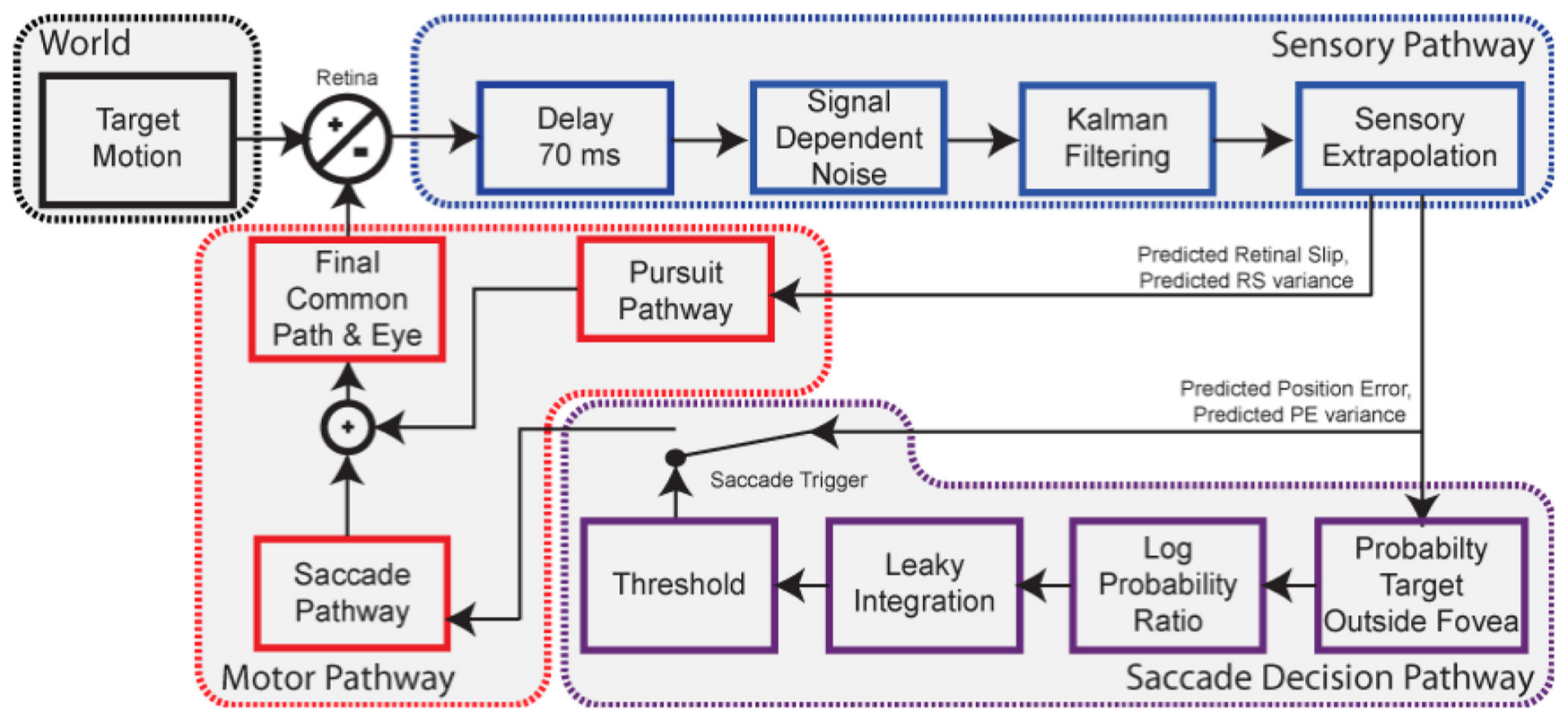

Figure 1. Saccade trigger model overview. A block diagram overview of the Bayesian decision model for saccade triggering. The sensory pathway estimates noisy signals from the world and extrapolates those to predict future errors. The decision pathway estimates confidence in future error prediction and triggers a saccade for a certain confidence threshold. The motor pathway adds the pursuit and saccade motor commands. Adapted with permission from Coutinho et al. (2018).

(Krauzlis and Lisberger, 1994a). When significant position error (PE) is accumulated, a catch-up saccade may be triggered to re-foveate the target. Thus, accurate tracking requires a synergistic coordination of saccades and smooth pursuit eye movements to overcome retinal position and velocity mismatches, respectively.

For a long time, it was believed that saccades and smooth pursuit were controlled by independent functional and anatomic systems in the brain (Robinson, 1986). As a consequence, the properties of saccades and smooth pursuit were studied independently. The sensory inputs of pursuit were thought to consist only of velocity and acceleration (Krauzlis and Lisberger, 1994b). Likewise, saccades and their properties were thought to be strictly governed by position inputs (Wurtz and Optican, 1994). However, accurate saccades executed during pursuit challenged this notion, as both position and velocity error must somehow be accounted for in the saccadic system (de Brouwer et al., 2001). Similarly, the smooth pursuit system takes position inputs into account (Blohm et al., 2005; Schütz and Souto, 2011). Consequently, our current understanding describes saccades and pursuit as two outcomes of a synergistic sensorimotor process,

This work is supported by Natural Sciences and Engineering Research Council of Canada and Canada Foundation for Innovation.

Acknowledgements: We thank Michael Lewis for technical assistance. We also thank all study participants for their time in completing these long tasks.

Correspondence should be addressed to Omri Nachmani at omri.nachmani@queensu.ca.

https://doi.org/10.1523/ENEURO.0196-18.2019

Copyright (C) 2020 Nachmani et al.

This is an open-access article distributed under the terms of the Creative Commons Attribution 4.0 International license, which permits unrestricted use, distribution and reproduction in any medium provided that the original work is properly attributed. sharing sensory inputs, anatomic pathways, and functional regulation (Orban de Xivry and Lefèvre, 2007).

The decision to trigger a catch-up saccade is a tradeoff between tolerating a PE when no saccade is triggered or a transient loss of vision during the saccade due to saccadic visual suppression (Schiller, 1986). Thus, when should a saccade be triggered? A double-step ramp paradigm developed by de Brouwer et al. (2002a) allows us to systemically investigate catch-up saccade behavior as a function of position and velocity errors during initiation and maintenance of smooth pursuit. A ramp in target position generates an initial retinal slip (RS), while a step in target position generates an initial PE. de Brouwer et al. (2002b) demonstrated that catch-up saccades were less likely to occur when the expected time-to-foveate the target using pursuit alone [eye-crossing time $\left(T_{x e}\right)$, -PE/ RS] is between 40 and 180 ms into the future, which was referred to as the smooth zone. However, $T_{x e}$ is a deterministic measure, providing no mechanistic explanation for the trigger mechanism or how to account for sensory or motor uncertainty. Similarly, early models describe the trigger decision for a stationary saccade as a linear rise to threshold using the LATER model but do not address sensorimotor context of the target or ongoing smooth pursuit (Carpenter and Williams, 1995; Reddi and Carpenter, 2000). As such, a re-examination of the saccade trigger is needed in this new context.

We have recently developed a Bayesian decision model that offers a mechanistic explanation of the catch-up saccade trigger (Fig. 1).

Our model relies on probabilistic estimation of predicted PE ( $\left.P E_{\text {pred }}\right)$ through Kalman filtering, i.e., an estimate with uncertainty due to sensory noise. This probabilistic estimate of $\mathrm{PE}$ is transformed into an esti- 
mate of confidence that a target is outside the fovea. Analogous to the evidence accumulation of a LATER model, confidence rises as sensory evidence is accumulated, rising faster with larger estimates of $\mathrm{PE}_{\mathrm{pred}}$ and/or lower uncertainty. Thus, the occurrence and time to trigger of the saccade is determined if and when this confidence estimate reaches threshold for saccade trigger.

Our current study aims to answer how PE and RS inputs are used by human subjects to make a trigger decision. The classical index thought to trigger catch-up saccades is a deterministic $T_{x e}$. Throughout the study, we compare the explanatory power of $T_{x e}$ to our probabilistic trigger mechanism based on future error. We hypothesized that the saccade trigger mechanism is an uncertainty dependent decision-making process that utilizes prediction of future sensory inputs. Using a wide range of moving target parameters to induce catch-up saccades during steady-state pursuit, we will be testing the following novel qualitative predictions that our model makes (rationale in the analysis plan): (1) large pre-saccadic $\mathrm{PE}_{\mathrm{p}^{-}}$ reds from target motion lead to less variable and shorter saccade trigger times; (2) target step followed by motion towards the fovea will produce highly variable as well as long saccades trigger times, whereas motion in the opposite direction will produce low variability and short saccade trigger times; (3) applying a Gaussian blur to the target will increase uncertainty in $\mathrm{PE}_{\text {pred }}$ and thus increase variability in saccade trigger times; and (4) for a given crossing time, large velocity steps (VSs) accrue larger PEs and thus lead to more occurrences of catch-up saccades with short trigger times. Null hypothesis: saccade triggering is determined by $T_{x e}$, independently of $\mathrm{PE}_{\text {pred }} \mathrm{S}$, where negative or large positive crossing times trigger saccades.

\section{Materials and Methods}

This study is a quantitative analysis study. Eye movement data are collected from subjects and analyzed to reveal the presence or absence of predicted patterns of behavior (see above predictions). No experimental blinding was involved in this study since eye movement control is stereotyped and catch-up saccade triggering appears to be automatic, often unconscious, and therefore beyond cognitive control. There was no existing data in our lab pertaining to smooth pursuit catch-up saccade triggering. While our study produced similar data to de Brouwer's catch-up saccade study, this dataset was recorded with modern video eye-tracking techniques, involved new experimental conditions and designs, and the analysis was guided by our model to uncover the functional mechanism leading to saccade triggering (as opposed to de Brouwer's purely descriptive analysis).

\section{Pre-registration}

This study was pre-registered at eNeuro as a registered report. The purpose of registered reports is to minimize biases in deductive science by pre-registering the proposed hypotheses of the experiment. The hypotheses, methods, and analysis plan of a registered report are reviewed before the research being conducted to ensure sound scientific reasoning. Another goal of pre-registration is to promote open science. As such, the regis- tered report is available on the open-science framework (OSF), the code necessary to run and analyze the experiment is available on GitHub, and the raw and labeled dataset is available on Dryad, links provided. Registered report OSF link: https://osf.io/wvjbf/; task and analysis code GitHub link: https://github.com/BlohmLab/SaccadeTrigger; data repository Dryad link: https://doi.org/ 10.5061/dryad.245j1p8.

\section{Sampling plan}

This study focused on automatic, stereotyped behavior and all participants exhibited similar patterns of behavior. A power analysis based on effect sizes from the model concluded that the minimum number of participants need is $N=12$. We recruited 15 participants (seven female) from the neuroscience department who were unfamiliar with the double-step ramp task. Eligible subjects had normal or corrected-to-normal vision. Each participant completed 10 data collection sessions lasting $\sim 45 \mathrm{~min}$ with 10 blocks each consisting of 50 trials per block, yielding a total of 5000 trials per participant and 75,000 trials altogether.

\section{Experimental procedure}

A double step-ramp task (de Brouwer et al., 2002a) generated with MATLAB (MathWorks, Inc) using the Psychophysics Toolbox (Brainard, 1997) was displayed on a ViewPixx (VPixx Technologies, $120-\mathrm{Hz}$ refresh rate, resolution $1920 \times 1200)$ screen positioned $50 \mathrm{~cm}$ from the participants spanning $60^{\circ}$ of their visual field. All trials began with an initial fixation target $\left(0.5^{\circ}\right.$, black) positioned $20^{\circ}$ to the left or right of the visual field followed by an abrupt position step (PS) away from the center $\left(2^{\circ} / 4^{\circ} / 6^{\circ}\right)$ with consecutive VS $\left(10^{\circ} / 20^{\circ} / 30^{\circ} / \mathrm{s}\right)$ toward the center to induce steady-state smooth pursuit. After a variable timeperiod (500-700 ms), a second step-ramp occurred in either direction. The VS was randomly chosen from a uniform distribution ranging from $-50 \%$ to $50 \%$ s. PSs were selected $\left(-20^{\circ}\right.$ to $\left.20^{\circ}\right)$ based on the VSs to limit the absolute time-to-foveate (-PS/VS) to under $1000 \mathrm{~ms}$. Target velocities and displacements varied randomly between each step-ramp and trial which ensured that the target jumps remained unpredictable. Each recording session alternated between clear and blurred target conditions. The purpose of the blurred target condition was to observe the influence of additional sensory noise on behavior. In the blurred target condition, the target was a 2D Gaussian blur $\left(50^{\circ}, \sigma=5^{\circ}\right)$. Half of the participants began with the blurred condition and half with the clear condition. Eye movements were tracked using an EyeLink (SR Research EyeLink 1000) video-based eye tracker with a sampling rate of $1000 \mathrm{~Hz}$. Position signals were low-pass filtered by a zero-phase digital filter (cutoff: $50 \mathrm{~Hz}$ ). Velocity and acceleration were derived from position signals using a central difference algorithm and saccades were detected using an acceleration threshold of $750 \% \mathrm{~s}^{2}$. All data of the target and eye movement trajectories and timing was stored on the computer during the experiment to be used for offline analysis. To minimize measurement error, participants performed calibration and validation tasks on blocks 1, 4, and 7. Data collection began only if 


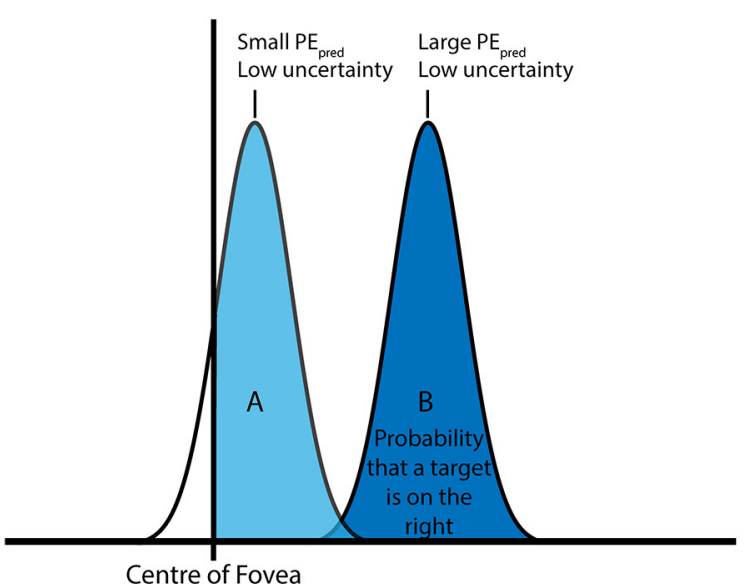

B A

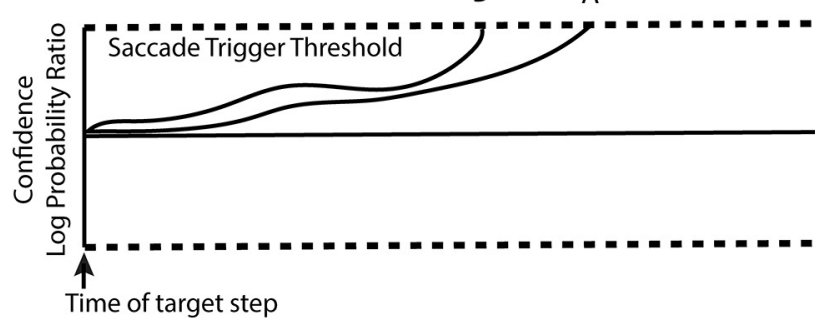

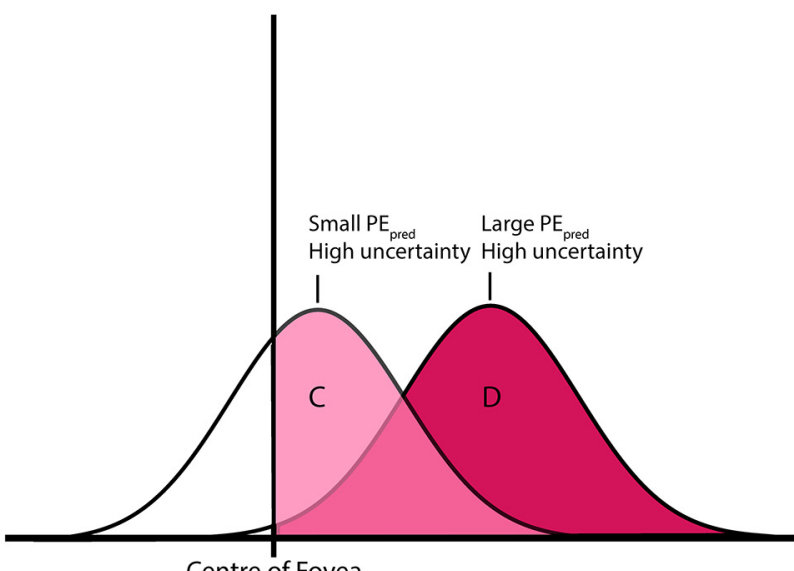

Centre of Fovea

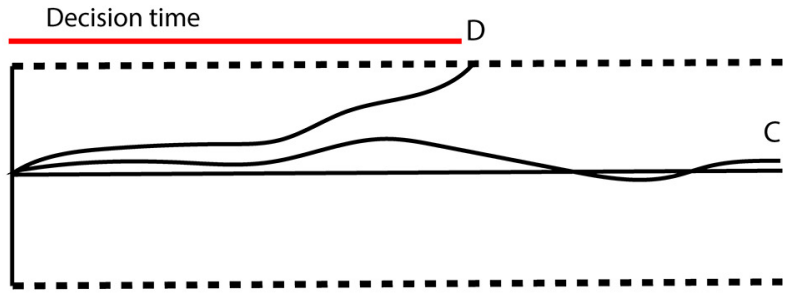

Figure 2. Model illustration of saccade trigger decisions. A representation of the interaction between PE magnitude, uncertainty, and the confidence that the target is outside the fovea. The confidence that the target is to the right of the fovea is represented by the ratio of the AUC to the right of fovea and the left of the fovea (Fig. 2, top panels). Saccades are triggered when the confidence signal rises to threshold (Fig. 2, bottom panel).

the calibration error obtained from EyeLink was below 0.2 on the $x$-axis relevant to the task.

\section{Pre-processing}

Individual trials were visually inspected for errors. Trials were discarded from the analysis if participants clearly did not track the target (e.g., due to a distraction), if blinks occurred during or after the second double-step (our critical period), if eye tracking data were missing (e.g., pupil detection failed), or if a saccade occurred during the second double-step (because visual processing would be disrupted). The remaining trials were filtered to remove occurrences of double saccades, as their execution and main sequence differed from the rest of the data, and saccades occurring after the second target step but made to the trajectory of the first target ramp. The total percentage of trials discarded was $23 \%$, yielding $\sim 58,000$ trials for analysis.

\section{Computations on data}

We computed instantaneous $\mathrm{PE}_{\text {pred }}$ over time as follows: $P E_{\text {pred }}$,

$$
P E_{\text {pred }}(t)=P E(t)+d t * R S(t),
$$

where $\mathrm{PE}$ is the difference between target and eye positions (target-eye) and RS is the difference between target and eye velocities (target-eye). The variable dt is the extrapolation duration and is typically between 90 and 290 ms (Robinson, 1986; de Brouwer et al., 2002b). By default, we used $\mathrm{dt}=150 \mathrm{~ms}$ to extrapolate PE as used by our model. Our model predicted that a saccade will be triggered if $\mathrm{PE}_{\text {pred }}$ is significantly different from zero, assuming a certain measurement noise.

Target-crossing time $\left(T_{x t}\right)$ is a measure of how much time it will take for a target to re-cross its initial trajectory following a step in position and velocity. This measure is agnostic to any behavioral measurements as it only relates to target motion dynamics. $T_{x e}$, on the other hand, measures the time it will take for the eye to contact the target given the current position and velocity error. At the moment of target step, $\mathrm{T}_{\mathrm{xt}}$ and eye-crossing are comparable in magnitude. $T_{x t}$ and $T_{x e}$ were computed as follows: $\mathrm{T}_{\mathrm{xt}}$,

$$
T_{x t}=-P S / V S
$$

and $T_{x e}$,

$$
T_{x e}=-P E / R S,
$$

where PS is position step, VS is velocity step, PE is position error, and RS is retinal slip.

The trigger mechanism in our model relies on confidence, i.e., the probability that the target is outside the fovea [area under the curve (AUC) in Fig. 2, top panel].

Incoming sensory input is noisy and has a level of uncertainty that depends on the sensorimotor context (SD in Fig. 2). Thus, when PE is small (Fig. $2 A, C$, top panel), 
higher uncertainty can considerably reduce the confidence that the target is to the right of the fovea. When the magnitude of $\mathrm{PE}$ is high (Fig. $2 B, D$ ), variations in uncertainty do not significantly affect the confidence that the target is to the right of the fovea. When this confidence reaches a threshold (Fig. $2 A, B, D$, bottom panel), a catch-up saccade is triggered to the peak of the probability density function. The confidence signal for lower PEs with high uncertainty fails to reach threshold and will not trigger a saccade (Fig. $2 \mathrm{C}$, bottom panel). The decision threshold is a tuned model parameter describing the amount of accumulated confidence necessary to trigger saccades.

Model simulations of two step-ramp trials demonstrate the evolution of sensory estimates as evidence accumulates and the variance from the Kalman filter decreases (Fig. 3).

The rate of rise to threshold determines the saccade trigger time. "Red" trials show large and increasing predicted errors with low uncertainty and thus the rate of rise to trigger saccades is higher. In the "blue" trial, confidence in predicted error never reaches threshold and a saccade is not triggered (Fig. 3).

Small but sustained predicted errors with low uncertainty will produce a slower rise to threshold and ultimately trigger a late saccade. If the predicted error is very large, uncertainty will have little influence on the rate of rise and a saccade will be triggered quickly.

\section{Pre-registered hypotheses}

Hypothesis 1

Small pre-saccadic $\mathrm{PE}_{\text {pred }} \mathrm{s}$ from target motion lead to highly variable and longer saccadic trigger times.

To test this hypothesis, $\mathrm{PE}_{\text {pred }}$ was calculated continuously (Eq. 1) for all trials for each individual participant. Trials were then sorted into saccade trials and smooth trials based on whether a saccade occurred within $400 \mathrm{~ms}$ of the second target step. For saccade trials, $\mathrm{PE}_{\text {pred }}$ for each trial was averaged over a $50-\mathrm{ms}$ window from target step. Trials were then sorted into $5^{\circ}$ bins of increasing magnitude in $\mathrm{PE}_{\text {pred }}$ with the corresponding saccade trigger time (saccade onset time - target step time) for each trial. We then calculated the saccade trigger time median and interquartile range (IQR) for each bin. Median and IQR were compared across participants using a repeated measures one-way ANOVA analysis. To further support our hypothesis and avoid commonly encountered issues with the frequentist approach to analysis, data were also examined by estimating the Bayes Factor (Wagenmakers, 2007). We expected a significant difference between saccade trigger time medians and variability in the low versus high $\mathrm{PE}_{\text {pred }} \mathrm{S}$.

\section{Hypothesis 2}

Target step followed by motion toward the fovea (foveopetal) will produce highly variable and longer saccade trigger times, whereas motion in the opposite direction will produce low variability and short saccade trigger times.

The direction of the position or VS can also significantly influence saccade trigger times. For example, using
$P E_{\text {pred }}$ (Eq. 2), a PS of $5^{\circ}$ and VS of $10 \%$ produces a $P E_{\text {pred }}$ of $6.5^{\circ}$ while the same PS with a VS of $-10 \%$ s produced a $P E_{\text {pred }}$ of $3.5^{\circ}$. Both $P E s$ may require a saccade, however, in the former the target is continuously moving away from the fovea, while in the latter the $P E_{\text {pred }}$ is decreasing. We can further capture this by calculating the $T_{x t}$ for target motion (Eq. 1). In the former situation, $T_{x t}$ $=-500 \mathrm{~ms}$, negative, which requires an immediate saccade as the target's distance from the fovea is increasing, whereas in the latter, $T_{x t}=500 \mathrm{~ms}$, positive, the decision to trigger a saccade is not as obvious as the target is approaching the eye and a participant may wait to accumulate more evidence. We expect to see more variability in trigger times in saccades occurring in the positive $T_{x t}$ range and more narrow trigger time distributions in the negative $T_{x t}$ range (Fig. 4, top panels).

To test this, $T_{x t}$ was calculated (Eq. 1) for all trials for individual participants and the corresponding saccade trigger time (target step time - saccade onset time) for that trial was obtained. Trials of individual participants were then sorted into three bins according to their $T_{x t}\left(T_{x t}\right.$ $<0,0<\mathrm{T}_{\mathrm{xt}}<400, \mathrm{~T}_{\mathrm{xt}}>400$ ). Individual participant's trigger time distributions for each bin were calculated and compared across participants using a repeated measures one-way ANOVA with $\mathrm{T}_{\mathrm{xt}}$ bin as a factor. Post hoc comparisons were performed using paired $t$ tests with familywise corrected error rates. We then calculated the Bayes factor using the sum of squares from the ANOVA analysis. We expected to see narrow trigger time distributions for negative $T_{x t}$ and wider trigger time distributions for positive and large $T_{x t}$.

\section{Hypothesis 3}

Applying a Gaussian blur to the target will increase uncertainty in $\mathrm{PE}_{\text {pred }}$ and thus increase variability in saccade trigger times.

We hypothesized that increase in uncertainty of $P E_{\text {pred }}$ due to a blurry target can lead to higher variability of saccade trigger times (Fig. 4). To test this, we compared trials in the clear condition with those in the blur condition. We again obtained trigger time distributions for the two conditions and compared the median and IQR of saccades in the $P E_{\text {pred }}$ and three $T_{x t}$ using a two-way ANOVA analysis and additional Bayes factor estimation. We expected to see more variability in the blurred target condition than in the clear target condition

\section{Hypothesis 4}

For a given $T_{x t}$, large VSs accrue larger PEs and thus lead to more occurrences of catch-up saccades with shorter and less variable trigger times.

Our model predicted that contrary to de Brouwer's findings, the smooth zone is not static but rather decreases in size with increasing VS. At higher VSs, pursuit velocity is typically below target velocity. This may lead to larger PEs and therefore larger $P E_{\text {pred }} s$ and more saccades (Fig. 5, top panel). Our model also predicted that target VS size will significantly change the trigger time distributions (Fig. 5, bottom panel)

We compared trials where saccades occurred within $400 \mathrm{~ms}$ of target step and those where no saccades were 
A

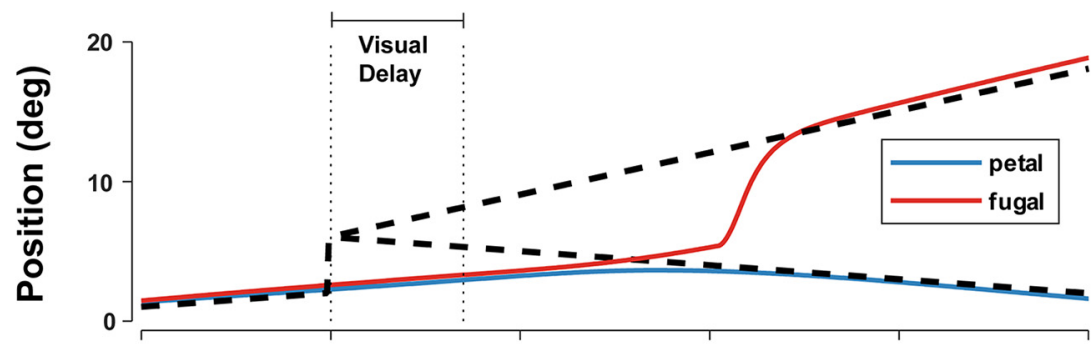

B

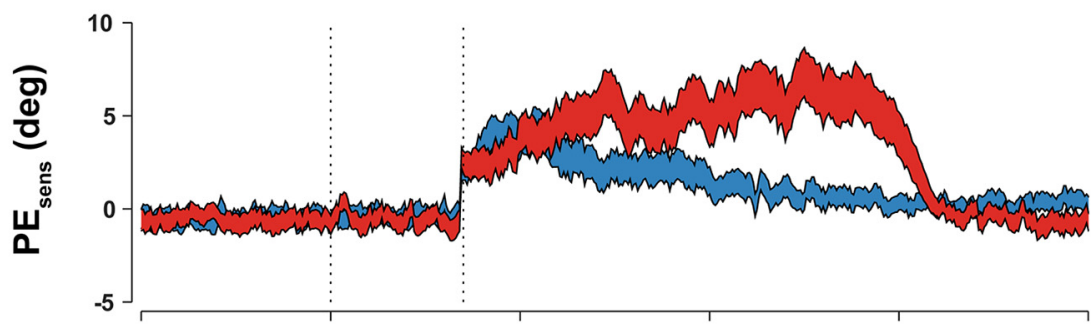

C

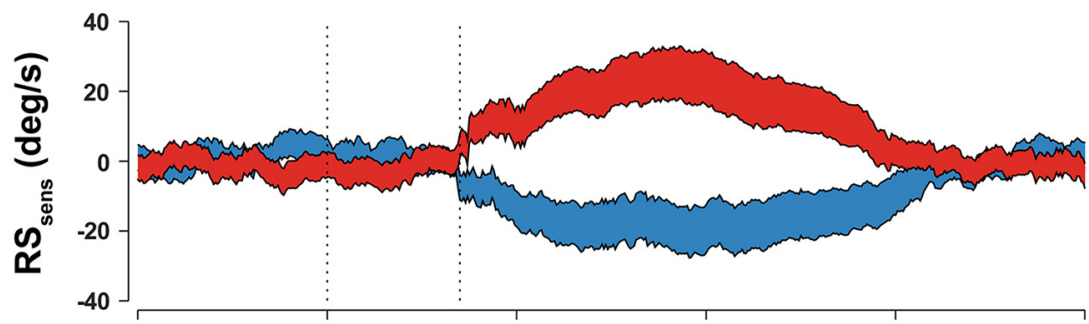

D

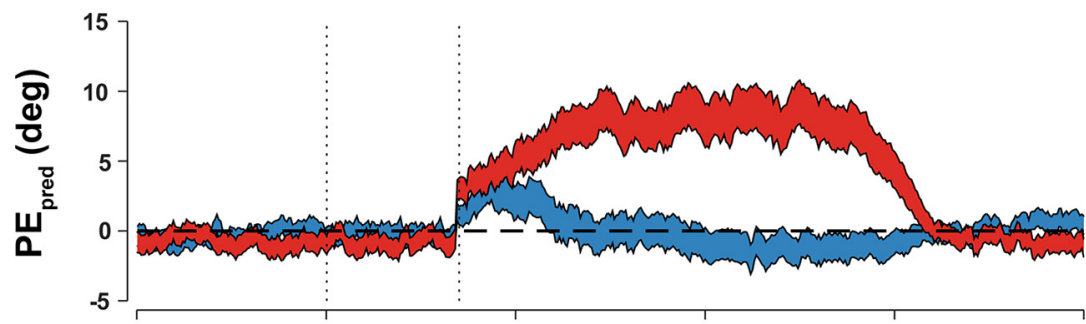

E

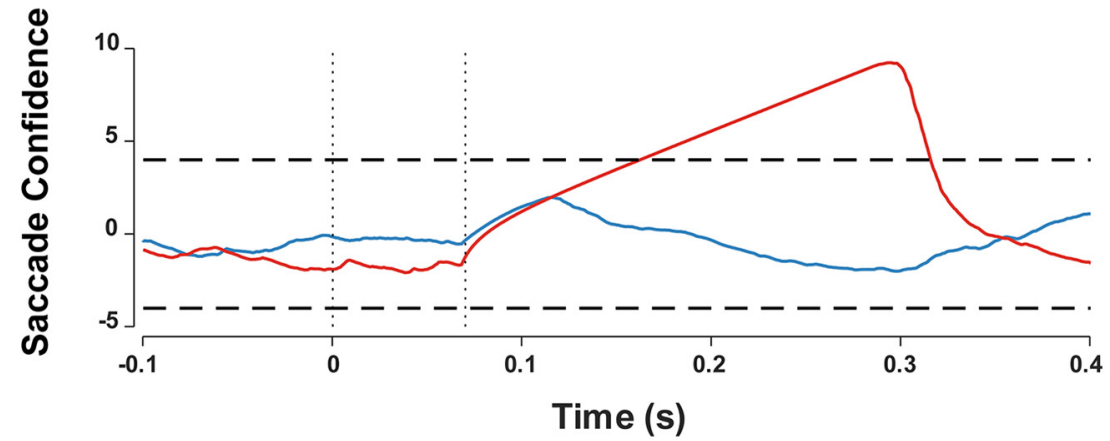

Figure 3. Single trial model simulations. $\boldsymbol{A}$, Target motion that results in a saccade and another that results in smooth pursuit with no saccade. $\boldsymbol{B}, \boldsymbol{C}$, Noisy PE and RS sensory signals are estimated continuously using a Kalman filter. $\boldsymbol{D}$, Future PE is continuously estimated using sensory signals. $\boldsymbol{E}$, A rise to threshold using a log probability ratio or confidence of sensory evidence leads to a trigger or no trigger decision.

observed in that time window. All trials were sorted into five equally spaced bins according to their absolute second VS $(0-50 \%)$. Trials were then sorted once more according to their $\mathrm{T}_{\mathrm{xt}}$ as in the previous analysis. We then used two-way ANOVA to compare the proportion of sac- cade trials and their trigger time in three VS conditions $\left(0-10 \%\right.$ s, $20-30 \%$ s, $40-50 \%$ s) and each $\mathrm{T}_{\mathrm{xt}}$ bin to observe whether the increase in RS led to a narrower smooth zone, shorter saccade trigger times, and narrower distributions. We estimated the Bayes factor and performed 

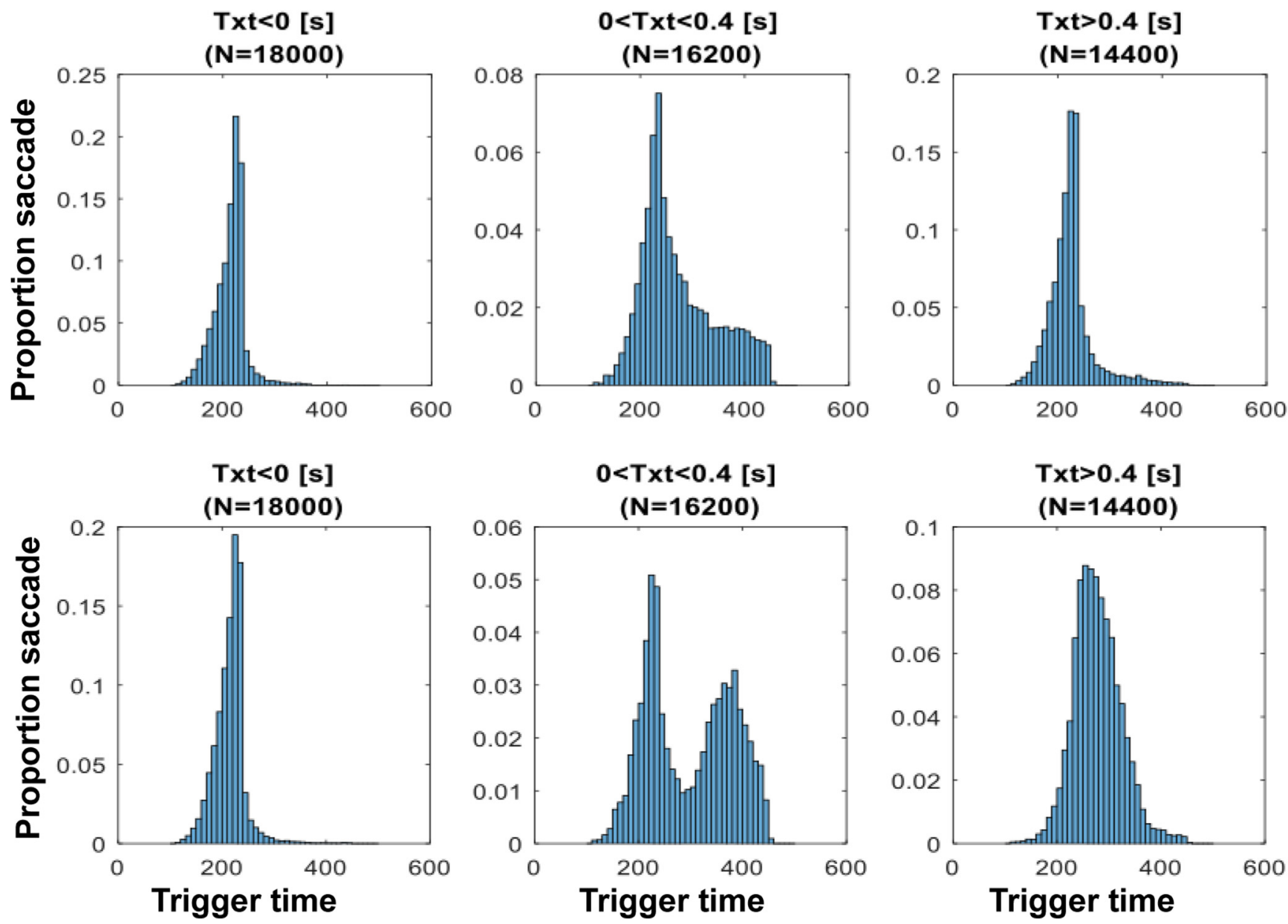

Figure 4. Model simulations of trigger time distributions. Model generated saccades trigger time distributions shown made to a clear target (Fig. 4, top panels) and a blurred target (Fig. 4, bottom panels). When the target is moving away from the fovea ( $\left.T_{x t}<0\right)$, uncertainty in $\mathrm{PE}$ does not significantly change trigger time distributions. For $\mathrm{T}_{\mathrm{xt}}>0$, increasing uncertainty in $\mathrm{PE}$ using blurred target increases saccade variability, especially around the smooth zone $\left(0<\mathrm{T}_{\mathrm{xt}}<400\right)$.

post hoc comparisons using paired $t$ tests with familywise corrected error rates.

\section{Null hypothesis}

Saccades are triggered when time-to-foveation (-PE/ RS) is smaller than $40 \mathrm{~ms}$ or larger than $180 \mathrm{~ms}$ as described by de Brouwer et al., 2002b.

For reproducibility, we compared our analyzed data collected from our "clear" condition to the analysis from de Brouwer et al., 2002b.

For all analyses, we concluded that differences in saccade trigger times are significant if the unrounded $p$ value from our ANOVA analysis (with conservative Bonferroni correction for multiple comparisons) was smaller than 0.05 .

\section{Results}

Our study aimed to recast former proposals for the triggering of catch-up saccades. The first section of our results (Figs. 6-Figs. 8) reintroduces the double-step ramp paradigm and sensory phase plots as a means to explore overall behavioral trends of catch-up saccades, with a novel addition of a side-by-side comparison of typical saccade trials and their corresponding temporal evolution on the phase plot. The purpose of this section is to highlight the similarities in behavioral trends of saccade triggering to previous work while also pointing out some explanatory gaps that motivated this current study. Next, we demonstrate how $\mathrm{PE}_{\text {pred }}$ satisfactorily explains both the occurrence of catch-up saccades and the saccade trigger time (Figs. 9, 10). Data presented in the figures provide comparisons between clear and blurred target conditions to observe the effects of sensory uncertainty. Additionally, all data are computed using both $\mathrm{PE}_{\text {pred }}$ and the previously proposed $T_{x e}$ or $T_{x t}$. These comparisons intend to demonstrate the superior explanatory power of $\mathrm{PE}_{\text {pred }}$ computation in the trigger mechanism. We then present trigger time distributions, summary bar graphs, and statistics in support of our pre-registered hypotheses (Figs. 11, 12). We conclude by exploring an interesting finding from the trigger time distributions (Figs. 13, 14) and provide final thoughts on the nature of the saccade trigger mechanism (Fig. 15).

\section{Temporal evolution of sensory inputs}

The trigger system for catch-up saccades depends on position and velocity sensory inputs. As such, in this 

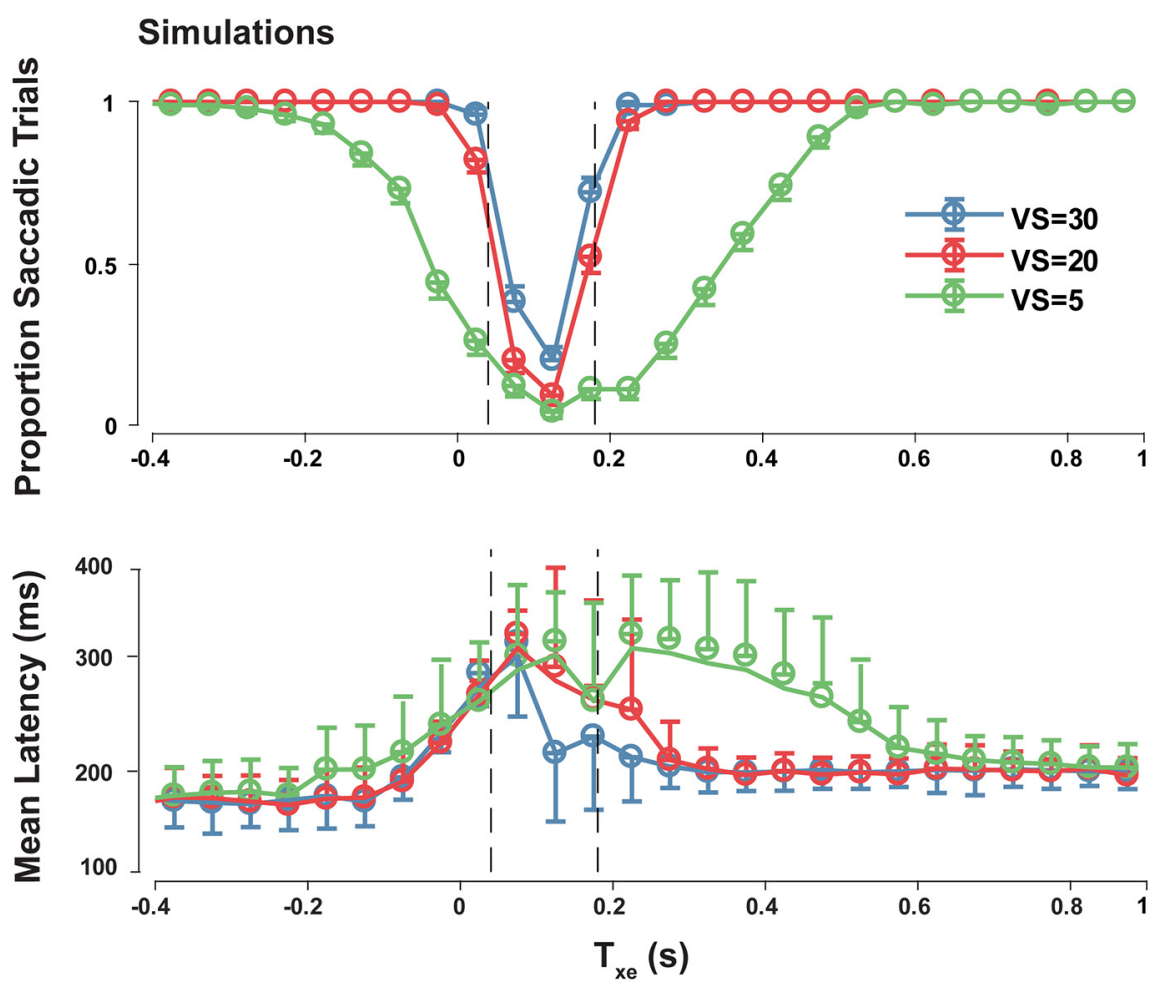

Figure 5. Model simulation trends of saccade trial proportions and trigger times. Model simulations of proportion of saccade trials and the trigger times of saccade trials compared to $T_{x e}(-P E / R S)$ for different VSs. The smooth zone boundaries from de Brouwer et al. (2002b) are marked by vertical dashed lines. The proportion of saccades near the smooth zone increases with increasing target VS. The saccade trigger times decrease with increasing target VS.

section we explore how varying these inputs changes saccade and smooth pursuit behavior. The step-ramp paradigm had been widely used in behavioral eye movement studies as a tool to induce smooth pursuit tracking with minimal catch-up saccades. As subjects fixate on a foveal target, the target abruptly steps peripherally by two to six visual degrees while simultaneously moving toward and past its previous location. If the target re-crosses the fovea in a short amount of time (100-200 ms), the eye responds by slowly accelerating in the same direction as target movement, rather than triggering a saccade to the peripheral target location. During steady state pursuit of the target, visual delays and imperfect gain can lead to the eye gradually falling behind the target, resulting in RS, a mismatch of target and eye velocity. Consequently, RS is accompanied by PEs, a mismatch between target and eye position. If sustained and large enough, both PE and RS lead to a catch-up saccade. To investigate under which conditions a catch-up saccade is triggered during sustained pursuit, we introduced a second step in both target position and velocity with a wide range of values (Fig. 6).

Following this additional random step in target position or velocity, the evolution of PE or RS are highly dependent on the motion dynamics of the target and pursuit ability of the subject. For example, when a PS occurred in the positive (rightward) direction and a VSs occurred in the negative (leftward) direction, or vice versa, PE naturally decreased as the target crossed its original trajectory. Conversely, if both position and VSs occurred in the same direction, PE continued to increase from the original PS.
These dynamics are further explored in the typical trials in Figure 7, left panels.

In a typical trial, a subject may trigger several saccades to track the target. Of interest is the first saccade triggered after the second target step, since the system was perturbed with specific and controlled position and VSs. The range of behaviors observed can be classified into three categories: early saccades, late saccades, and smooth trials where no saccade occurred for the first 400 ms (de Brouwer et al., 2002b). In Figure 7, left panels, late saccades such as blue and "turquoise" often result from a failure to estimate or match target velocity, as the eye anticipates crossing but eventually moves past the clear target. Importantly, in the blurred condition, subjects demonstrate comparable foveal tracking during sustained pursuit. Clear red, "green," blurred "yellow," and "pink" represent targets moving foveofugally (away from fovea) after the target step, whereas the rest of the trials move in the foveopetal (toward fovea) direction.

To observe overall behavioral trends of saccade occurrences under different sensory conditions, we plotted a sensory phase plot (Fig. 7, right panels) of the instantaneous PEs and RSs $100 \mathrm{~ms}$ before saccade onset, thought to be the last instant that sensory information can influence saccade decisions to provide enough time for saccade programming and execution (Orban de Xivry and Lefèvre, 2007). The double coned region with a visibly lower density of saccade occurrence is referred to as the smooth zone (de Brouwer et al., 2002b). This region is more evident under the blurred condition, as higher un- 
A
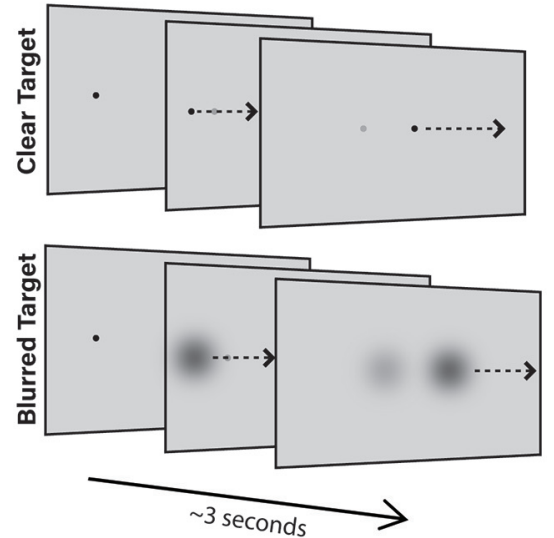

C

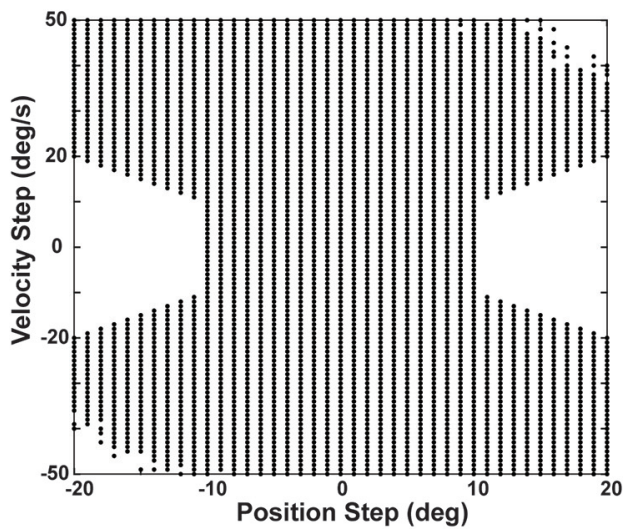

B

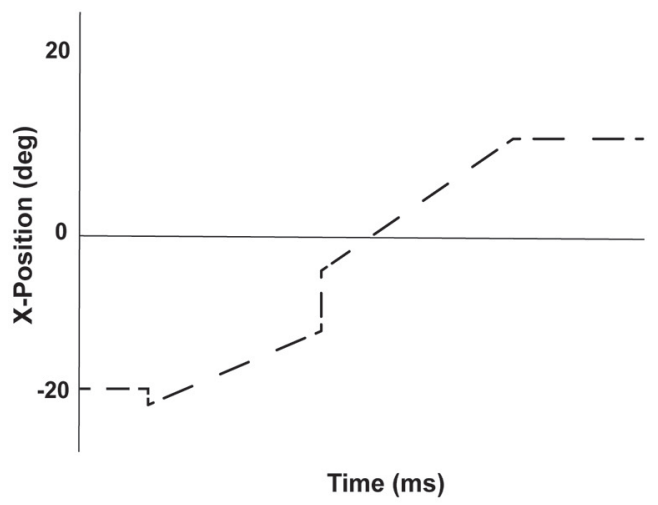

D

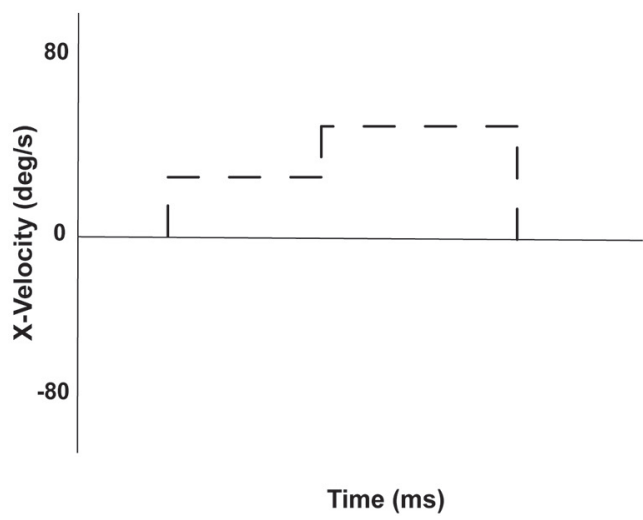

Figure 6. Double step-ramp task. A, Representation of the double step ramp task on a screen. Each participant completed ten alternating sessions of clear and blurred target conditions. Each session consisted of 10 blocks of 50 trials each. $\boldsymbol{C}$, Parameter range for PSs and VSs used to induce catch-up saccade triggering. Large steps were omitted as they resulted in off-screen targets. $\boldsymbol{B}$, $\boldsymbol{D}$, Position, velocity-time graphs of the tracking dot for a typical trial. Shown are a positive PS (to the right) and a positive VS (rightward acceleration).

certainty led to more smooth trials. The ratio of PE and RS that defines the slope boundaries of this zone is defined as the $T_{x e}$ from 40 to $180 \mathrm{~ms}$, although it is immediately evident that many saccades are still triggered within the smooth zone. Furthermore, of interest to us was how such sensory inputs evolve temporally from the moment of target step, depicted by the overlaid colored traces. Two high density regions of saccades are apparent on either side of the " 0 PE." These regions result from late saccades such as "orange" and blue that move into the smooth zone but fail to match target velocity and thus trigger a saccade as soon as they cross the target. Short saccade trigger times such as red or green tend to start far from and continue to move away from the smooth zone.

Figure 8 shows continuous sampling of PE and RS for trials where no saccades occurred in the first $400 \mathrm{~ms}$, classified as smooth trials. Successful smooth trials result when participants accelerate or decelerate to match target velocity and minimize both PE and RS to $\sim 0$. This region of dense smooth trials corresponds to the low saccade density zone in the Figure 7.

Together, the traces in Figures 7, 8 provide intuition for how sensory inputs evolve through time and lead to a saccade trigger, although on their own, they are only descriptive of behavioral correlates. Moreover, the smooth zone boundaries and the corollary $\mathrm{T}_{\mathrm{xe}}$ index poorly explain several trends in the data. For example, some trials that either briefly cross or even remain in the smooth zone nevertheless trigger a saccade. Conversely, many smooth trials begin far outside the smooth zone and proceed toward it. The variability in behavioral observations contradicts previously established notions of an absolute decision criteria of saccade trigger based on $\mathrm{T}_{\mathrm{xe}}$ and suggests a probabilistic explanation driven by statistical decision theory. In this view, noisy sensory estimates of PE and RS are continuously sampled and used to make predictions of future PE. In turn, these predictions are used to make motor decisions to trigger or not to trigger a catch-up saccade. Importantly, high uncertainty in the prediction will delay or suppress saccade triggering.

\section{$\mathrm{PE}_{\text {pred }}$ explains saccade occurrence}

We hypothesized that any decision signal that leads to a catch-up saccade must reach a decision threshold $\sim 100 \mathrm{~ms}$ before saccade onset, to allow time for saccade preparation and motor execution. Therefore, we sampled PE and RS 100 ms before the first catch-up saccade of each saccade trial and computed $\mathrm{PE}_{\text {pred }}$ using Equation 1, 

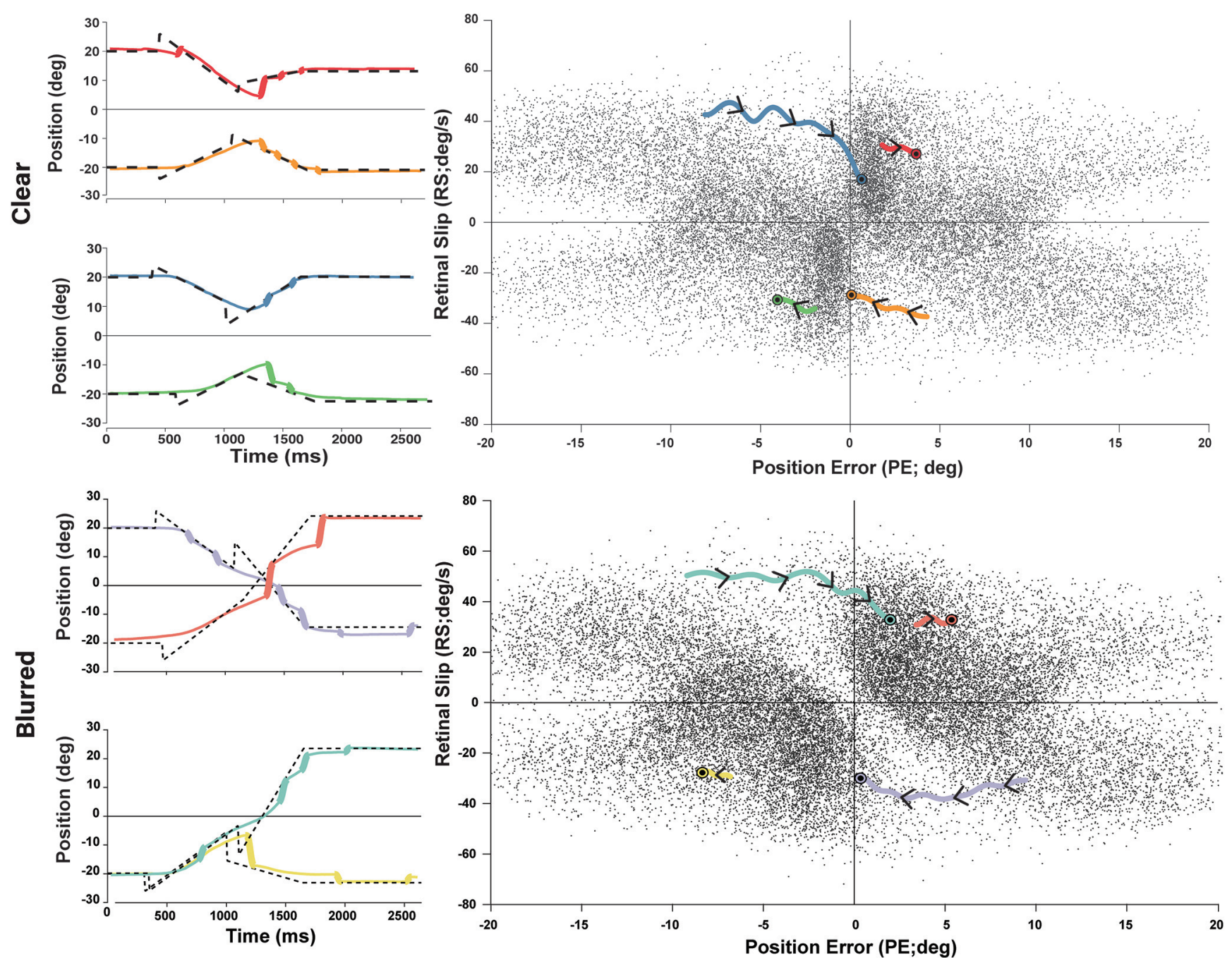

Figure 7. Sensory phase plot and typical saccade trials. For each saccade trial, PE and RS were sampled 100 ms before saccade onset and plotted on a phase plot. Left, Four typical trials for clear and blurred target conditions plotted in pairs on a position-time graph, with saccades in bold. Right, PE and RS phase plots of all trials for clear and blurred target conditions. The colored traces correspond to the evolution of sensory inputs of the typical trials on the left from the target step to 100 ms before saccade onset (represented by black circles). Note that when PE and RS have opposite signs, PE naturally decreases and when PE and RS have the same sign, PE naturally increases in absolute value.

as well as $T_{x e}$ using Equation 3. For smooth trials, we averaged PE and RS over the initial $400 \mathrm{~ms}$ following second target step. In Figure 9, saccade trial proportions are plotted as a function of both $\mathrm{PE}_{\text {pred }}$ and eye crossing time for different magnitudes of VSs.

The large dip in saccade occurrence from centered at 0 $\pm 5^{\circ}$ for $P E_{\text {pred }}$ and $100 \pm 100 \mathrm{~ms}$ for $T_{x e}$ has been referred to as the smooth zone. The boundaries of the smooth zone are far more sensitive to differences in VS when plotted by $\mathrm{T}_{\mathrm{xe}}$ compared to $\mathrm{PE}_{\text {pred }}$, likely due to the instability of the $T_{x e}$ signal as velocity errors approach zero, as RS is in the denominator. Figure 9A,C shows near $100 \%$ saccade trials outside the $-5^{\circ}$ to $5^{\circ}$ range, consistent with the hypothesis that $\mathrm{PE}_{\text {pred }}$ plays a central role in the saccade trigger decision. As anticipated (Fig. 9A) larger VSs lead to higher signal-dependent noise (SDN) and thus lower proportion of saccades. Interestingly, higher sensory uncertainty under the blurred target condition notice- ably reduces the influence of VS related SDN and also significantly reduces saccade proportions, suggesting that noise from a blurred target is a larger driver of uncertainty and strongly modulates motor decisions.

\section{$\mathrm{PE}_{\text {pred }}$ and uncertainty modulate saccade trigger time}

Further exploring how well $\mathrm{PE}_{\text {pred }}$ explains the trigger decision, we investigated the effects of $P E_{\text {pred }}$ on trigger time, which was defined as the time from target step to saccade onset. To make inferences on how the magnitude of $P E_{\text {pred }}$ influences trigger time, $P E_{\text {pred }}$ was sampled at target step and averaged over $50 \mathrm{~ms}$ from target step. Similarly, at the time of target step, $T_{x t}$ (Eq. 2) was used in place of $T_{x e}$ in accordance with previous work. Figure $10 A, C$ shows that trigger times increase as $\mathrm{PE}_{\text {pred }}$ approaches zero, and comparably higher VS lead to longer trigger times due to higher SDN. 

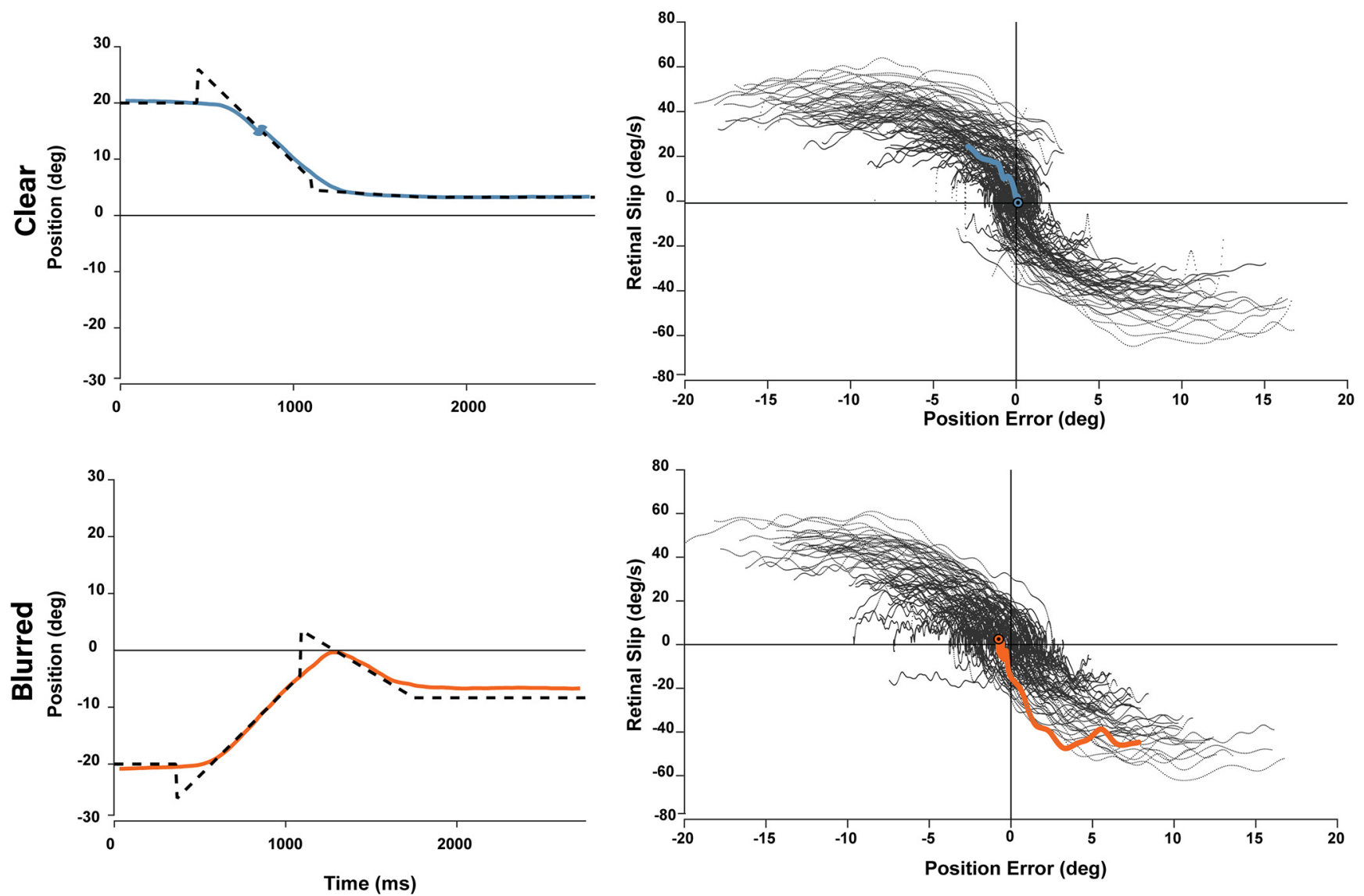

Figure 8. Sensory phase plot and typical smooth trials. For smooth trials where no saccade occurred within $400 \mathrm{~ms}$ of target step, $\mathrm{PE}$ and RS were sampled continuously and plotted on a phase plot. Left, Typical smooth trial on a position-time graph where eye traces are colored. Trials are considered smooth if no saccades occur within the first $400 \mathrm{~ms}$ after target step. Right, Phase plot showing continuous sampling of PE and RS for a subset of trials. Smooth trials tend to begin in the upper left or lower right corners where PE naturally decays as PE and RS are in opposite directions. The colored traces correspond to the typical trials on the left, showing the evolution of PE and RS toward the center where near-perfect tracking is achieved. The red dotted traces represent the smooth zone boundaries from de Brouwer et al. (2002b).

Under high uncertainty conditions (Fig. 10C) trigger times increase considerably only for small $\mathrm{PE}_{\text {pred }}$ but remain the same for very large $P E_{\text {pred }}$, supporting our model predictions (Fig. 2). Notably, in Figure 10B,D, although the $T_{x t}$ plots show similar patterns of longer trigger times surrounding the smooth zone, the trends are far noisier at lower VSs and large positive $\mathrm{T}_{\mathrm{xt}}$. Under preregistered hypothesis 4 , we predicted longer and more variable trigger times for smaller VSs for a given $T_{x t}$. $A$ two-way ANOVA revealed a significant interaction between VS and $T_{x t}$ medians $\left(F_{(2,14)}=5.791, \eta_{p}^{2}=0.293, p\right.$ $\left.=0.001, \mathrm{BF}_{10}=1.001 \mathrm{e}+35\right)$ but no significant interaction of IQRs $\left(F_{(2,14)}=1.064, \eta^{2}{ }_{p}=0.071, p=0.314, \mathrm{BF}_{10}=\right.$ $2.846 \mathrm{e}+20)$ in the clear target conditions. However, post hoc analysis revealed that smaller VSs lead to shorter and less variable trigger times only when $T_{x t}$ was positive $(p=$ 0.009), opposite to our prediction. This trend does not hold under blurred conditions as seen in Figure 10D. We conclude that although $T_{x t}$ influences trigger times, its effects were not predictable and consistent across different experimental conditions.

\section{Pre-registered hypothesis 1 and 3}

We hypothesized that targets with small $P E_{\text {pred }} s$ and high uncertainty produce highly variable and shorter saccade trigger times. As outlined in the pre-registration, $P E_{\text {pred }}$ was calculated continuously for each saccade trial, sampled at target step, and averaged over a 50-ms window. Trials were then sorted into three bins of $<-5^{\circ},-5$ to $5^{\circ}$, and $>5^{\circ} P E_{\text {pred }}$ bins with their corresponding saccade trigger times. Large negative $P E_{\text {pred }}$ was defined to be less than $-5^{\circ}$ and large positive $P E_{\text {pred }}$ defined to be $>5^{\circ}$. Small $P E_{\text {pred }}$ was defined to be between $-5^{\circ}$ and $5^{\circ}$. Means and standard deviations were not compared as the underlying trigger time distributions were non-Gaussian, therefore the medians and IQRs were more informative. Trigger time distributions for each bin were plotted for clear and blurred conditions (Fig. 11A-F). Median trigger times and IQR for each $P E_{\text {pred }}$ bin was calculated for each subject and compared across bins and conditions (Fig. $11 G)$.

There was a significant effect as determined by repeated measures one-way ANOVA between $\mathrm{PE}_{\text {pred }}$ magnitude and both median trigger time $\left(F_{(2,14)}=219.292, \eta^{2}\right.$ 

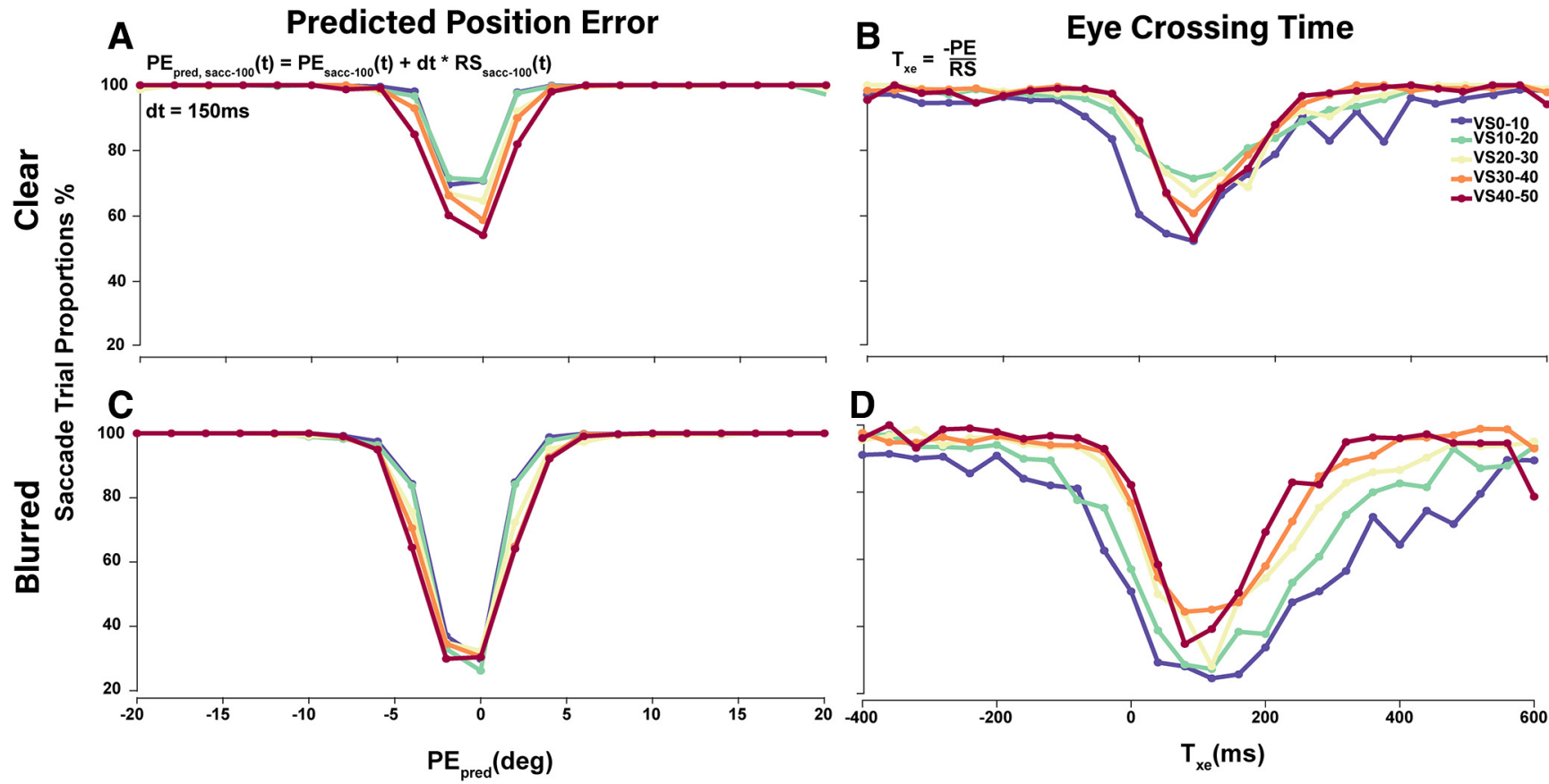

Figure 9. Proportion of saccade trials relative to $P E_{\text {pred }}$ and $T_{x e}$. $P E_{\text {pred }}, P E$, and $R S$ were sampled $\sim 100$ ms before saccade onset for saccadic trials and averaged over the first $400 \mathrm{~ms}$ after target step for smooth pursuit trials. $\boldsymbol{A}, \boldsymbol{C}$, Saccade trial proportions plotted for a given $\mathrm{PE}_{\mathrm{pred}}$ from $-20^{\circ}$ to $20^{\circ}$ in bins of $2^{\circ}$. $\boldsymbol{B}, \boldsymbol{D}$, Saccade trial proportions plotted for a given $\mathrm{T}_{\mathrm{xe}}$ from -400 to $600 \mathrm{~ms}$ in bins of $50 \mathrm{~ms}$.

$\left.=0.940, p=3.101 \mathrm{e}+14, \mathrm{BF}_{10}=1.190 \mathrm{e}+31\right)$ and IQR $\left(F_{(2,14)}=108.47, \eta_{\mathrm{p}}^{2}=0.886, p=5.6329 \mathrm{e}-8, \mathrm{BF}_{10}=\right.$ $5.230 \mathrm{e}+16)$. Confirming hypothesis 1 , a post hoc multiple comparison with Bonferroni correction revealed that small $P E_{\text {pred }}$ trigger times were significantly longer than large $\mathrm{PE}_{\text {pred }}$ trigger times $(+\sim 72 \mathrm{~ms}, p=1.2517 \mathrm{e}-9,5.720 \mathrm{e}+9)$
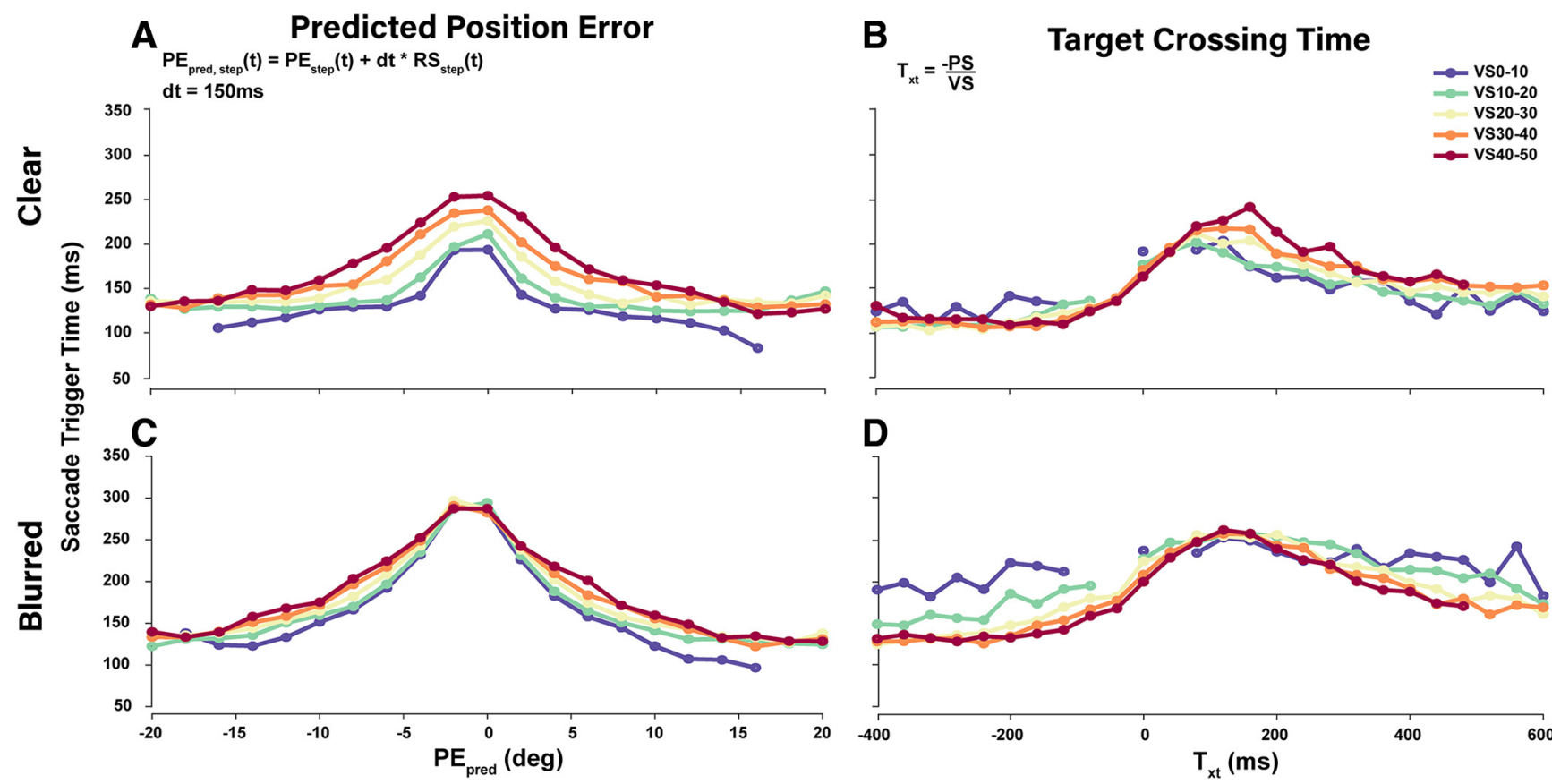

Figure 10. Collapsed saccade trigger time for ranges of $P E_{p r e d}$ and $T_{x t}$. Collapsed trigger time medians were plotted against $P E_{p r e d}$ and $T_{x t}$ for the relevant parameter ranges and increasing VSs. $\boldsymbol{A}, \boldsymbol{C}$, Collapsed Saccade trigger times as a function of PE different VSs. $\boldsymbol{B}, \boldsymbol{D}$, Collapsed saccadic trigger times as a function of $T_{x t}$ for different VSs showing an increase around values corresponding to the smooth zone. Missing data points for VS0-10 and VS10-20 result from PS being drawn from a uniform discrete distribution. 

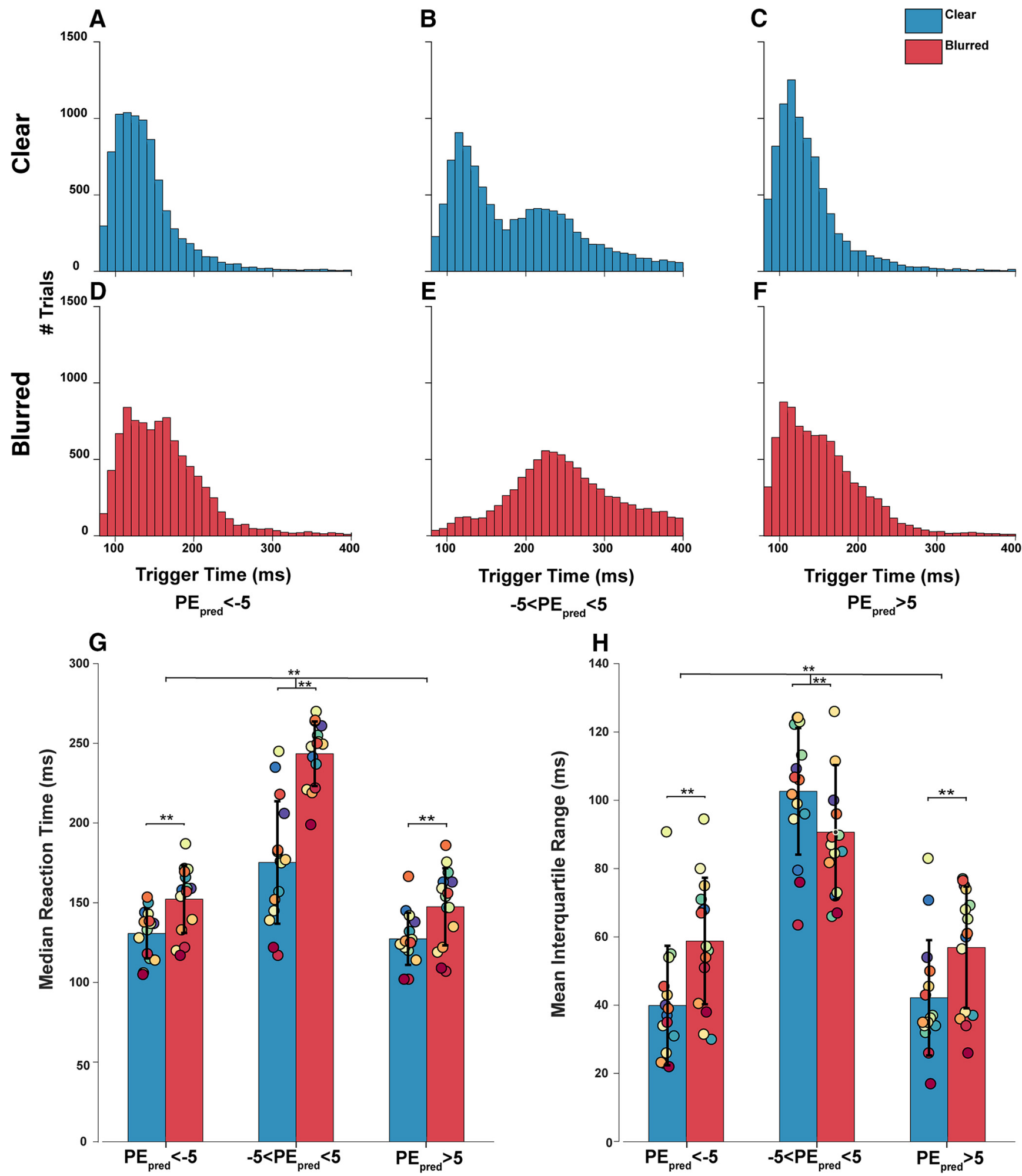

Figure 11. Saccade trigger time distributions and medians by $P E_{\text {pred. }}$ Saccade trigger times are measured from target step to saccade onset. to make conclusions on how $P E_{\text {pred }}$ contributes to saccade trigger time, $P E_{\text {pred }}$ here was also sampled from target step over $50 \mathrm{~ms}$ and averaged, rather than $\sim 100 \mathrm{~ms}$ before saccade onset. Trials were grouped into bins of large negative $(\boldsymbol{A}, \boldsymbol{D})$, small $(\boldsymbol{B}, \boldsymbol{E})$, and large positive $(\boldsymbol{C}, \boldsymbol{F}) \mathrm{PE}_{\text {pred }}$. $\boldsymbol{G}$, Collapsed median trigger times for all subjects for clear and blurred condition. $\boldsymbol{H}$, Collapsed IQR of all subjects for clear and blurred condition; ** represents a significant difference between groups with a $p<0.001$. Each colored dot represents a particular subject's mean value throughout all six bars. 
and had a larger IQR $\left(+\sim 47 \mathrm{~ms}, p=1.0421 \mathrm{e}-7, \mathrm{BF}_{10}=\right.$ $2.676 \mathrm{e}+7)$ compared to both negative and positive large $\mathrm{PE}_{\text {pred. }}$. There was no significant difference between median trigger time $\left(p=0.481, \mathrm{BF}_{10}=1.156\right)$ and IQR $(p=$ $\left.1.0, \mathrm{BF}_{10}=0.195\right)$ for negative and positive large $\mathrm{PE}_{\text {pred }}$ bins.

To observe the effects of a Gaussian blur on the trigger time distributions with respect to $P E_{\text {pred }}$ bins, a repeated measures two-way ANOVA was used to compare median trigger time and IQR of the same $P E_{\text {pred }}$ under clear and blurred target conditions. A Bayes two-way ANOVA was used to estimate the Bayes Factor. There was a statistically significant interaction between the effects of $P E_{\text {pred }}$ bins and target condition on median trigger time $\left(F_{(2,28)}=\right.$ $\left.30.043, \eta_{p}^{2}=0.682, p=0.000081, \mathrm{BF}_{10}=1.190 \mathrm{e}+31\right)$ and IQR $\left(F_{(2,28)}=8.237, \eta_{\mathrm{p}}^{2}=0.370, p=0.012, \mathrm{BF}_{10}=\right.$ $8.989 \mathrm{e}+18)$. Post hoc multiple comparisons confirmed Hypothesis 3 and revealed a significant increase in median trigger time $\left(+37 \mathrm{~ms}, p=4.4253 \mathrm{e}-8, \mathrm{BF}_{10}=\right.$ $3.668 \mathrm{e}+7)$ and weakly significant increase in IQR $(+7.2$ $\mathrm{ms}, p=0.029, \mathrm{BF}_{10}=0.85$ ) for all $\mathrm{PE}_{\text {pred }}$ bins with the addition of a Gaussian blur. Uncertainty most strongly modulated the trigger time when $\mathrm{PE}_{\text {pred }}$ was small $(+68$ $\mathrm{ms}$ ) but did not significantly increase the IQR, as shown in Figure 11.

The bimodality in Figure $11 B$ results from two underlying distributions of early saccades and late saccades (cutoff of $175 \mathrm{~ms}$ ) addressed later in this article. This bimodality largely disappears in Figure $11 E$ under blurred conditions, where the majority of trials are late saccade trials.

\section{Pre-registered hypotheses 2 and 3}

We hypothesized that saccades made to targets moving toward the fovea, as measured by $T_{x t}$, will have highly variable and longer trigger times. Trials were divided into bins of $\mathrm{T}_{\mathrm{xt}}$ (-Position step / Velocity step)with corresponding median trigger time and IQR for each subject and compared using repeated measures one-way ANOVA with post hoc comparison using Bonferroni correction, and a Bayes one-way ANOVA. The $T_{x t}$ bins were $<0 \mathrm{~ms}$, 0-400 ms, >400 ms. Positive $T_{x t}$ corresponds to foveopetal movement and negative $T_{x t}$ corresponds to foveofugal movement. Similarly, trigger time distributions for each bin were plotted for clear and blurred conditions (Fig. 12A-F). Median trigger times and IQR for each $P E_{\text {pred }}$ bin was calculated for each subject and compared across bins and conditions (Fig. 12G).

There was a significant effect as determined by repeated measures one-way ANOVA between median trigger time $\left(F_{(2,14)}=406.211, \eta^{2}=0.967, p=9.6867 \mathrm{e}-12\right.$, $\left.\mathrm{BF}_{10}=296.752\right)$ and IQR $\left(F_{(2,14)}=34.663, \eta_{\mathrm{p}}^{2}=0.712, p\right.$ $\left.=0.00004, \mathrm{BF}_{10}=1.692 \mathrm{e}+11\right)$. Post hoc multiple comparison with Bonferroni correction confirmed hypothesis 2 and revealed that trials with $T_{x t}$ between 0 and 400 had significantly higher median trigger time $(+76 \mathrm{~ms}, p=$ $9.8068 \mathrm{e}-12, \mathrm{BF}_{10}=1.783 \mathrm{e}+16 ;+57 \mathrm{~ms}, p=7.3585 \mathrm{e}-$ $\left.12, \mathrm{BF}_{10}=5.359 \mathrm{e}+13\right)$ compared to negative $\mathrm{T}_{\mathrm{xt}}$ and $\mathrm{T}_{\mathrm{xt}}$ $>400$, respectively, and significantly higher IQR $(+51 \mathrm{~ms}$, $p=2.0519 \mathrm{e}-8, \mathrm{BF}_{10}=1.492 \mathrm{e}+9 ;+25 \mathrm{~ms}, p=0.036$,
$\left.\mathrm{BF}_{10}=21.32\right)$. Negative $\mathrm{T}_{\mathrm{xt}}$ trials had a significantly lower median trigger time $\left(-18 \mathrm{~ms}, p=1.0 \mathrm{e}-5, \mathrm{BF}_{10}=593987\right)$ and IQR $\left(-27 \mathrm{~ms}, p=0.000316, \mathrm{BF}_{10}=135190.77\right)$ compared to trials with $T_{x t}>400$. A two-way ANOVA revealed weak statistically significant interaction between the effects of $T_{x t}$ bins and target condition on median trigger time $\left(F_{(2,28)}=5.070, \eta_{p}^{2}=0.266, p=0.041, \mathrm{BF}_{10}\right.$ $=1.100 \mathrm{e}+33)$ and significant interaction effects for IQR $\left(F_{(2,28)}=11.329, \eta_{\mathrm{p}}^{2}=0.447, p=0.005\right.$, BF $=$ $2.274 \mathrm{e}+13)$. Post hoc multiple comparisons revealed a significant increase in median trigger time $(+32 \mathrm{~ms}, p=$ 6.6819e-7, $\left.\mathrm{BF}_{10}=2.656 \mathrm{e}+10\right)$ and IQR $(+13 \mathrm{~ms}, p=$ $\left.0.001, \mathrm{BF}_{10}=51.76\right)$ in the Gaussian blur condition.

\section{Bimodal trigger time distributions of early and late saccades}

Thus far, both $P E_{\text {pred }}$ and $T_{x t}$ provide adequate explanations for the saccade trigger time, exhibiting similar distributions and effects in high uncertainty. This should come as no surprise as both metrics measure a property of target dynamics, future $P E$ being measured by $P E_{\text {pred }}$, while rate and direction of change of PE are captured by $\mathrm{T}_{\mathrm{xt}}$. The bimodal distributions seen in both Figures $11 \mathrm{~B}$, $12 B$ hint at two underlying distributions of early and late saccades, with a trigger time boundary of $175 \mathrm{~ms}$. Under high uncertainty, a shift in favor of late saccades occurs. To explore why, we classified all trials into early, late, or smooth categories using the 175-ms boundary and plotted trial proportions under different $\mathrm{PE}_{\text {pred }}$ and $\mathrm{T}_{\mathrm{xt}}$ conditions (Fig. 13).

These proportion plots explain the bimodality distributions in Figures 11, 12. In Figure $13 A, B$, when $\mathrm{PE}_{\text {pred }}$ was small (boxed region), the proportion of late trials was significantly higher when $T_{x t}$ is positive compared to when it was negative. In Figure $11 B$, the early peak corresponds to Figure $13 A$, while the late peak corresponds to Figure $13 B$. Blurred target conditions expand this zone and shift the proportions to favor late saccades, especially when $P E_{\text {pred }}$ was small (Fig. 13C,D, boxed region). Similarly, the 0 - to 400-ms region in Figure $13 E, F$ correspond to the bimodalities in Figure $12 B$. When $\mathrm{PE}_{\text {pred }}$ was small, the proportion of late trials was significantly higher compared to when $\mathrm{PE}_{\text {pred }}$ was large. Interestingly, under blurred conditions and small $\mathrm{PE}_{\text {pred }}$ (Fig. 13H), all $\mathrm{T}_{\mathrm{xt}}$ values from negative to positive resulted in high proportions of late and smooth trials. This unintuitive break in the pattern serves as a strong indication that $P E_{\text {pred }}$ rather than $T_{x t}$ is used as a decision signal for the catch-up saccade trigger mechanism, although information captured by $T_{x t}$ may still influence the rate of rise.

\section{$\mathrm{PE}_{\text {pred }}$ triggers catch-up saccades}

Lastly, for each trial type, we sampled and plotted $P E_{\text {pred }}$ and $T_{x t}$ as a scatterplot (Fig. 14). Under clear conditions, the majority of late and smooth trials appeared at small $P E_{\text {pred }}$ and positive $T_{x t}$. There was a strong boundary at the $0 \mathrm{~T}_{\mathrm{xt}}$, where the majority of smooth trials occur. When uncertainty was high however, the boundary disappeared and both late and smooth trials were dispersed along the $T_{x t}$ axis. In contrast, in both target conditions, the $0 \pm 10^{\circ}$ boundary of the $\mathrm{PE}_{\text {pred }}$ axis re- 

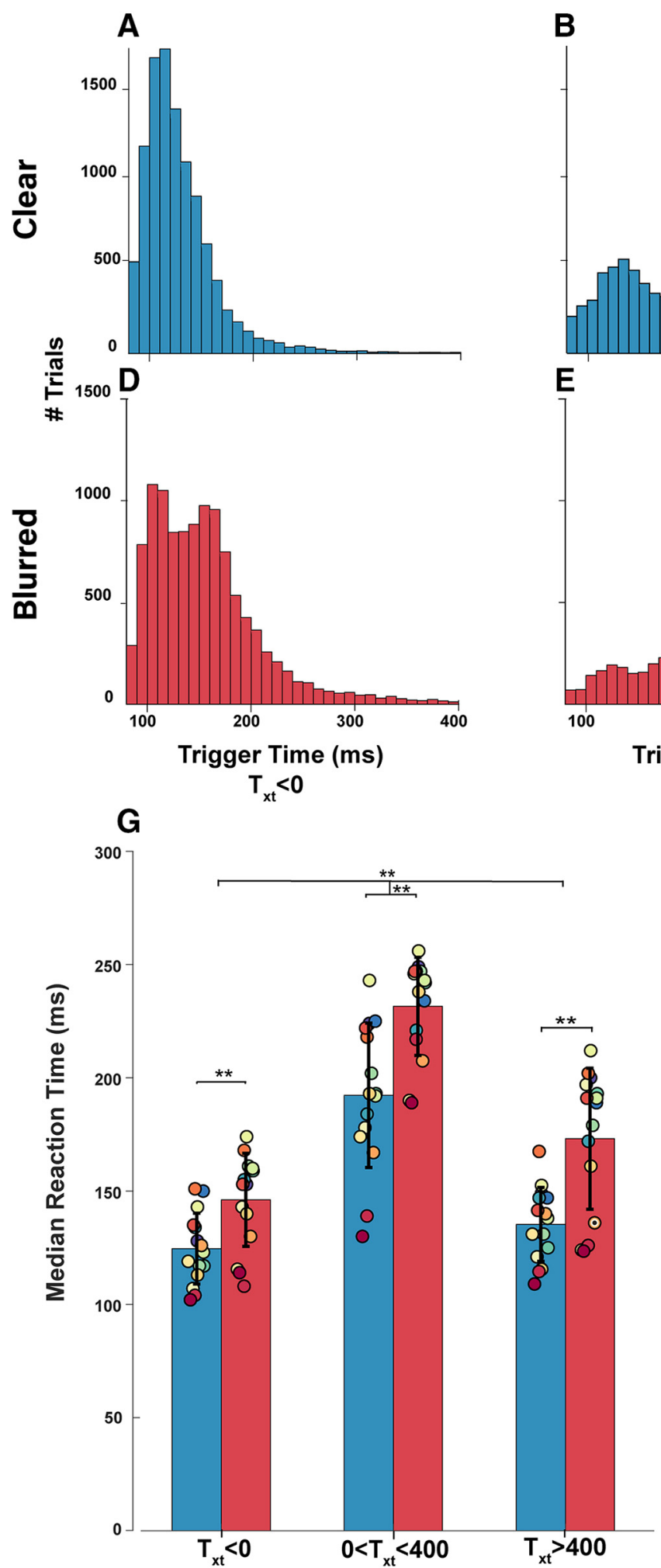
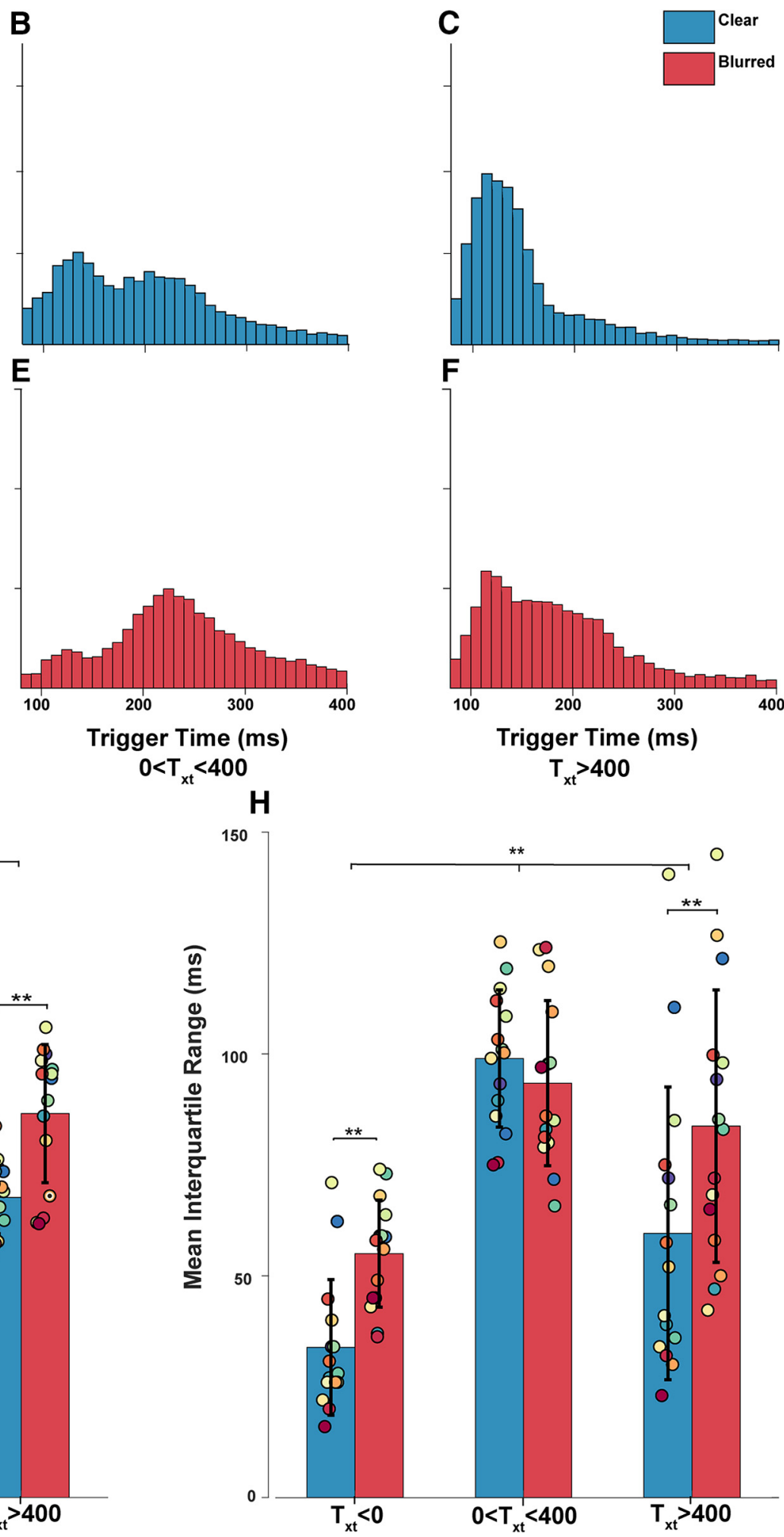

Figure 12. Saccade trigger time distributions and medians by $T_{x t}$. Similarly to Figure 5 , at target step, PE and RS can be approximated by $\mathrm{PS}$ and RS, and the index $T_{x t}$ is used to estimate time to contact and directionality. Positive $T_{x t}$ represents foveopetal motion and negative $T_{x t}$ represents foveofugal motion. Trials were grouped into bins of negative $(\boldsymbol{A}, \boldsymbol{D})$ small positive $(\boldsymbol{B}, \boldsymbol{E})$, and large positive $(\boldsymbol{C}$, $\boldsymbol{F}) \mathrm{T}_{\mathrm{xt}}$. The bimodalities in $\boldsymbol{B}, \boldsymbol{D}, \boldsymbol{E}$ represent distributions of early and late saccades, which will be addressed later in this paper. $\boldsymbol{G}$, Collapsed median trigger times for all subjects for clear and blurred condition. $\boldsymbol{H}$, Collapsed IQR of all subjects for clear and blurred condition; ** represents a significant difference between groups with a $p<0.001$. Each colored dot represents a particular subject's mean value throughout all six bars. 

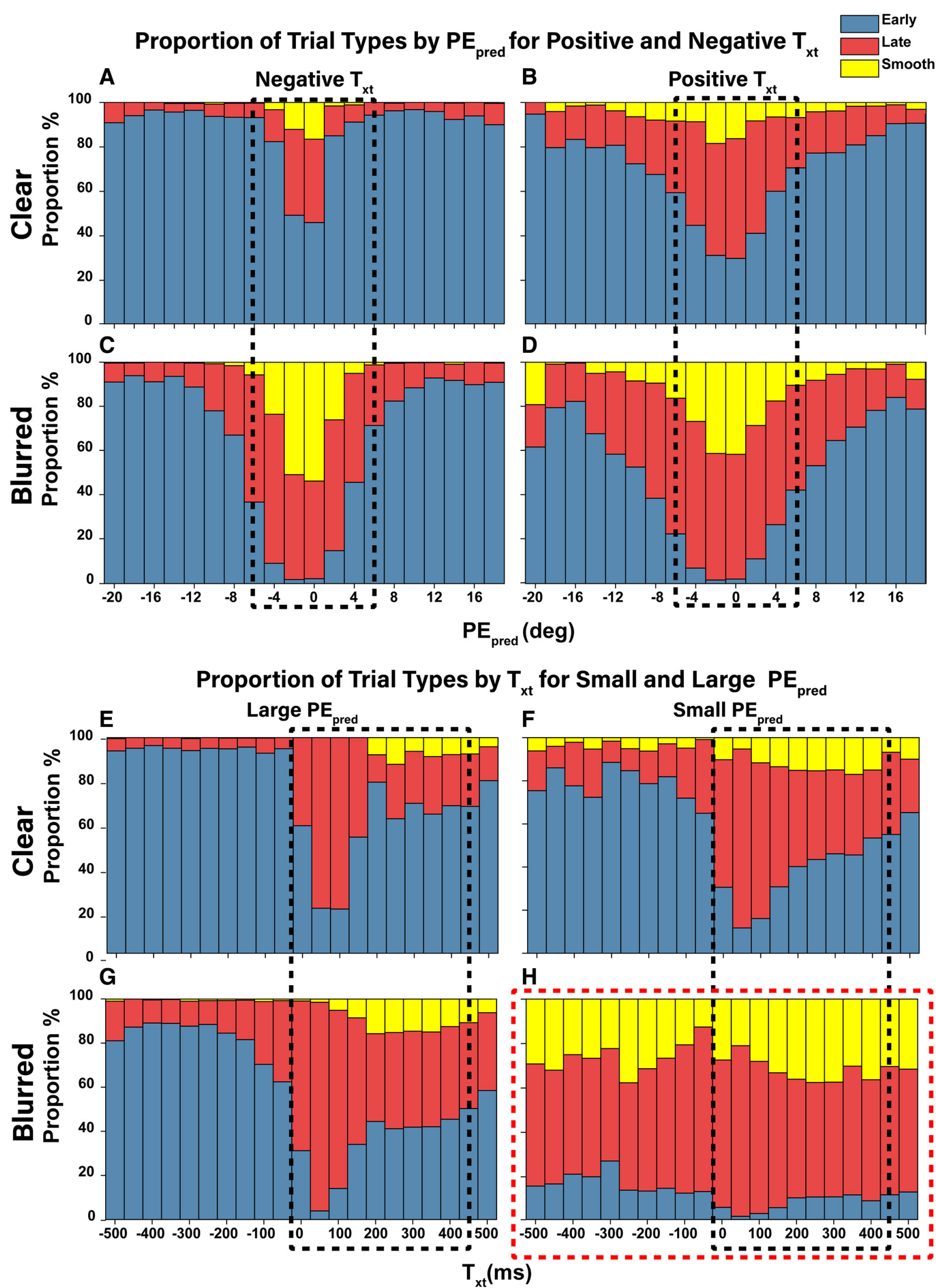

Figure 13. Proportion of early, late, and smooth trials under different $P E_{\text {pred }}$ and $T_{x t}$ conditions. Trials were classified as early, late, 
continued

or smooth based on the trigger time cutoffs of 175 and $400 \mathrm{~ms}$, as informed by trigger time distributions from Figures 5 . A-D, Variations of trial types by $\mathrm{PE}_{\text {pred }}$ bins under foveofugal and foveopetal $\mathrm{T}_{\mathrm{xt}}$. $\boldsymbol{E}-\boldsymbol{H}$, Variations of trial types by $\mathrm{T}_{\mathrm{xt}}$ bins under small and large $\mathrm{PE}_{\text {pred }}$.

mained. Trials outside the boundary were largely early saccade trials while those within the boundary were late or smooth saccade trials.

\section{Discussion}

\section{General discussion}

In this study, we investigated the trigger mechanism of catch-up saccades during sustained smooth pursuit. Our results provide strong support for the central role of $P E_{\text {pred }}$ in the trigger mechanism. As previous studies have shown, PE and RS are the primary sensory inputs that drive the saccadic-pursuit system (Orban de Xivry and Lefèvre, 2007). Suppression of saccade triggering is more probable when PE naturally decays due to RS in the opposite direction. We have further demonstrated that noisy estimates of PE and RS are likely used to predict future PE and make a trigger decision. Trials that generated predicted errors below $5^{\circ}$ frequently resulted in the suppression of saccade triggering or a triggering of a late saccade, whereas large predicted errors consistently triggered early saccades. Additionally, trigger time variability significantly increased when the preceding predicted error was small. By blurring the tracking target, we also demonstrated that high levels of sensory uncertainty strongly modulate the trigger decision. High uncertainty resulted in stronger saccade suppression when predicted errors were below $5^{\circ}$ but had no effect when predicted errors were large. High uncertainty also resulted in an overall increase of saccade trigger times, most significantly when predicted errors were small, although the effects on trigger time variability were weak. Saccades to targets moving foveopetally also resulted in longer and more variable trigger times, consistent with previous work (Bieg et al., 2015). Lastly, we observed significant trigger time increases due to both SDN from large RS and noise from high sensory uncertainty, further supporting a probabilistic explanation for the trigger decision. For each observation, the explanatory power of $\mathrm{PE}_{\text {pred }}$ was compared to the previously suggested metric of $\mathrm{T}_{\mathrm{xe}}$ or $\mathrm{T}_{\mathrm{xt}}$ (de Brouwer et al., 2002b). Overall, if in doubt of sensory inputs, the saccadic system suppresses the triggering of a catch-up saccade in favor of smooth pursuit. In comparison to $T_{x e} s$, explanations of behavioral data using $\mathrm{PE}_{\text {pred }}$ were more stable, intuitive, aligned with current literature (for review, see Shadmehr et al., 2010), and easily translatable to $2 \mathrm{D}$ or $3 \mathrm{D}$ tracking tasks where targets may not cross the initial point of fixation.

The smooth zone, as defined by $\mathrm{T}_{\mathrm{xe}}$, is tightly linked to our proposed predicted error mechanism. For example, consider Equation 1 in a situation when $P E_{\text {pred }}$ is 0 and a saccade is not needed. Rearranging this equation to solve for $d t$ returns Equation 3 for $T_{x e}$. In fact, our chosen $d t$ for linear extrapolation is $150 \mathrm{~ms}$, which is within the $T_{x e}$ smooth zone. However, $\mathrm{T}_{\mathrm{xe}}$ is a deterministic measure and does not include measures of uncertainty. Predicted error, however, accounts for uncertainty through the variance of the Kalman filtering estimate. At sudden stimulus changes, the variance of RS and PE is high; therefore, the probability distribution of having 0 predicted error is broad. As the variance decreases over time with better estimates, so does this distribution constrict around the true $P E_{\text {pred }}=0$ value (Fig. 15).

On the phase plot, a trial in the smooth zone can either move in the direction of the constricting boundary or opposite to it. In the former, the subject remains in the smooth zone for longer while in the latter, a participant exits the zone faster and triggers a saccade, resulting in high density saccade regions on either side of the $0 \mathrm{PE}$ boundary (Fig. 7). In this view, the effect of the blurred target condition will lead to higher position uncertainty and a wider decision boundary which result in higher saccade trigger times and smooth trials.

\section{Limitations and alternative interpretations}

An alternative interpretation of our results from the blurred target conditions is that participants were simply more tolerant of small PEs when the target was larger, as they could direct their gaze to a larger area. This is likely not true as the qualitative trends in both the clear and blurred target conditions are in agreement, and the observed quantitative differences match our hypothesized results. Another weakness in most eye-tracking studies, including this one, is that laboratory tracking tasks show little resemblance to tracking in the natural world. For example, the use of small foveal targets, uncommon in nature, may elicit saccades more frequently (Heinen et al., 2016). Accordingly, larger stimuli such as our blurred target may increase the tolerance for PEs rather than the sensory uncertainty, thus suppressing saccades and driving smooth pursuit. However, Melcher and Kowler (1999) demonstrated that regardless of stimulus shape, the saccadic landing position is computed as the center-of-area of that stimulus. Therefore, it is highly likely that for a given target location, the $\mathrm{PE}_{\text {pred }}$ for small or large targets will be the same.

The observed effects of saccade trigger times under different magnitudes of predicted errors could also partly be explained by whether attention allocation is ahead, or broadly ahead of a moving target (Khan et al., 2010; Chen et al., 2017), as attention will modulate the saccade trigger times. Additionally, a confound in our study is the heterogenous tracking ability of participants. Indeed, smooth pursuit performance predicts interception accuracy, strategy, and whether interception is early or late (Fooken et al., 2016). Thus, subjects who are naturally better at smooth pursuit such as those who play ball sports or video games may better correct for PEs using pursuit alone. Although some subjects were better smooth pur- 

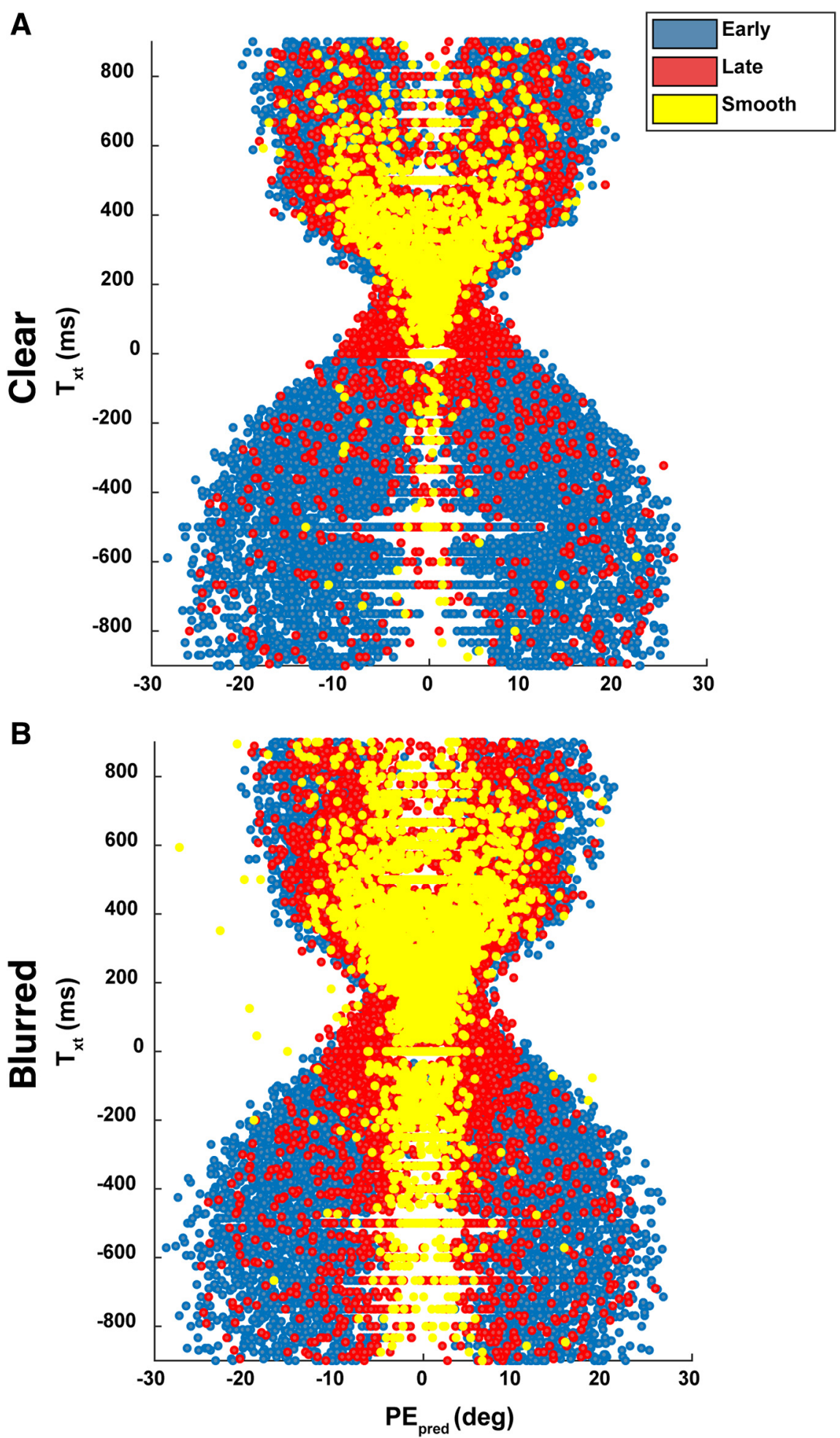

Figure 14. Individual trial scatterplot of $P E_{p r e d}$ and $T_{x t}$. To further illustrate the correlations between $P E_{p r e d}$ and $T_{x t}$, with saccade trigger times, single trials were plotted and assigned a color corresponding to their trigger time category. $\boldsymbol{A}$, Early saccades are evenly distributed along the parameter space, whereas smooth trials and late saccades are most evident for positive $T_{x t}$ and small $\mathrm{PE}_{\mathrm{pred}}$. $\boldsymbol{B}$, Blurred target conditions disperse late and smooth trial distributions as higher uncertainty reduces the likelihood for a saccade trigger.

suers, we observed similar trends across all subjects when results were plotted individually, in agreement with our overall hypotheses.
Furthermore, our results from Figures 13, 14 suggest that $T_{x t}$ is still relevant as it indicates whether predicted errors will continue to increase or decrease. Chang and 


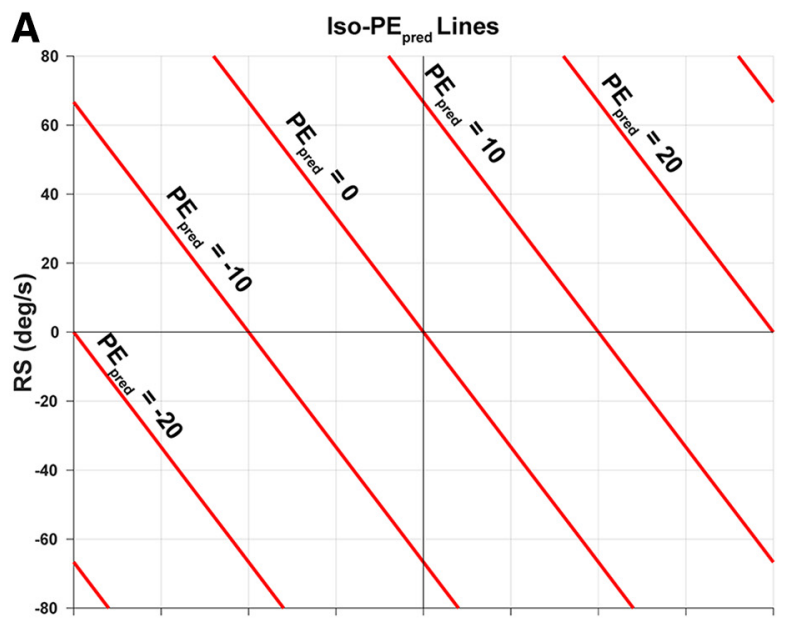

B Probability smooth shortly after step; High sensory variance

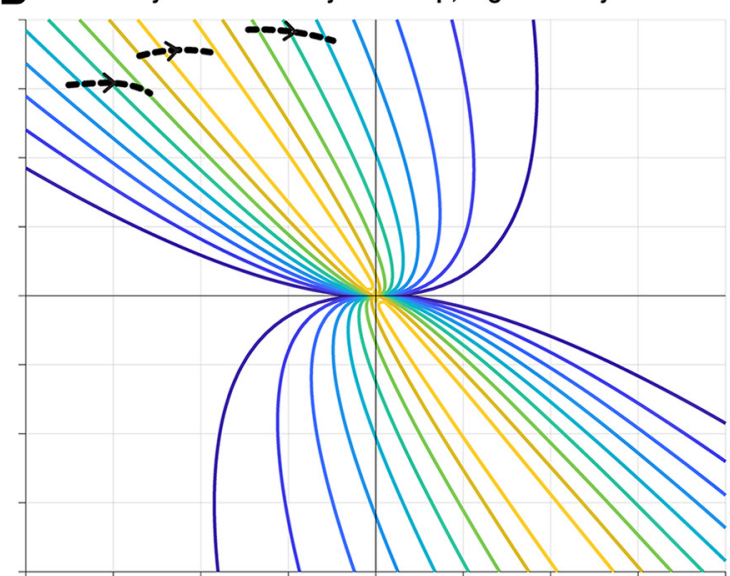

1

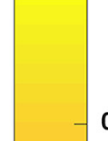

C Probability smooth $100 \mathrm{~ms}$ after step; Moderate sensory variance
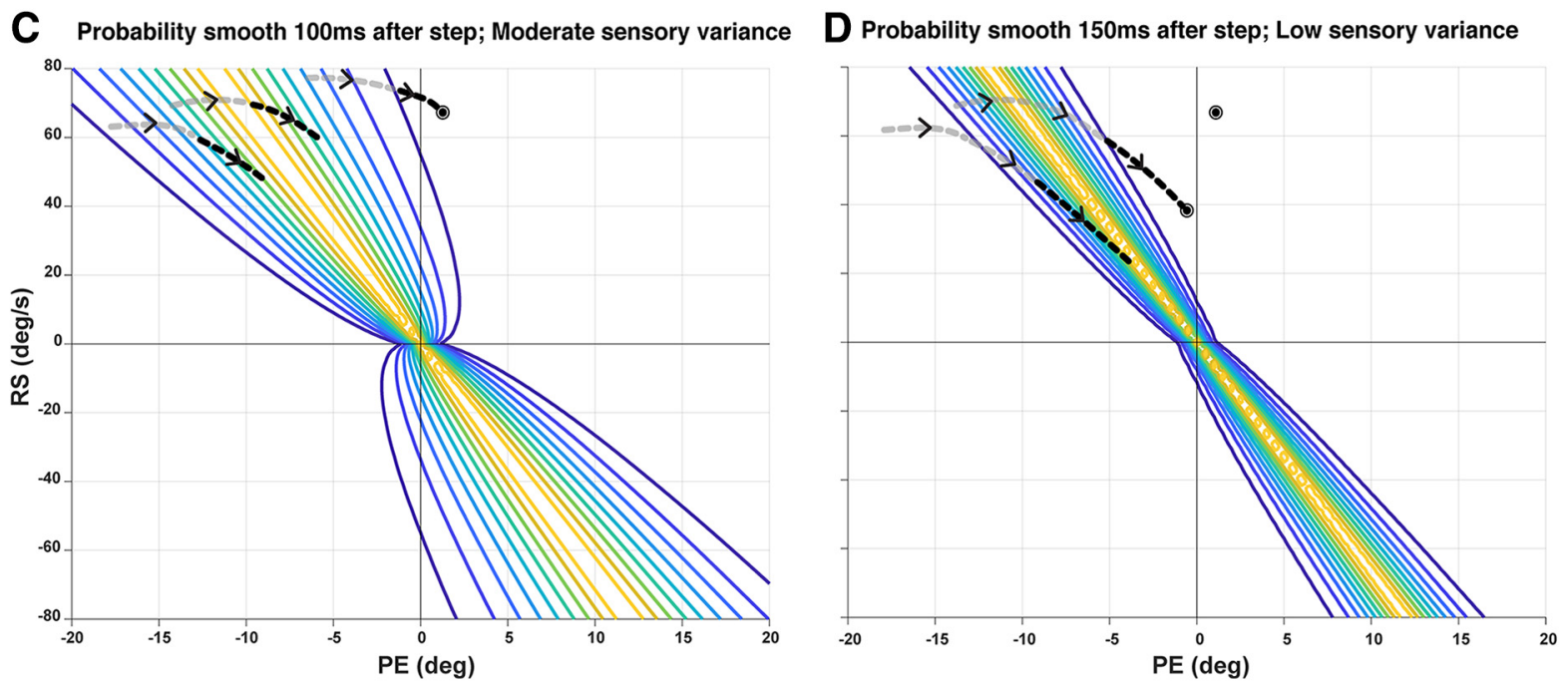

Figure 15. Illustration of a dynamic smooth zone. Visual illustration of how the width of the smooth zone or the decision boundary to trigger a saccade constricts as the variance of sensory estimation decreases over time. $\boldsymbol{A}$, Iso-PE $\mathrm{E}_{\text {pred }}$ lines on a phase plot where $P E_{\text {pred }}$ remains the same. Saccades are suppressed when predicted error is around $0 . \boldsymbol{B}$, Probability lines around $P E_{\text {pred }}=0$ shortly after the step where subjects should smoothly pursue the target without needing to trigger a saccade. This is where variance of sensory estimation of RS from Kalman filtering is highest. Three hypothetical temporal evolutions are shown as a visual aid. $\boldsymbol{C}$, Probability lines narrow slightly as evidence is accumulated and variance decreases. One trial exits the smooth zone and a saccade is triggered. $\boldsymbol{D}$, Probability lines are very narrow as the variance around RS estimates is small. Saccades will be triggered shortly after exiting this narrow zone. Another trial exits the smooth zone and a saccade is triggered. The remaining trial is still in smooth pursuit.

Jazayeri (2018) show that humans indeed exploit temporal information to make interception decisions. Perhaps humans combine information from both predicted errors and $T_{x t}$ to make the trigger decision. Lastly, some suggest that the superior colliculus is not involved in prediction as no neural correlates have been observed (Goffart et al., 2017). However, perhaps prediction is computed elsewhere in the brain and the necessary saccade amplitude is added to the superior colliculus motor command. Such mechanism would explain the asynchrony observed in the position and motion pathways of the saccadic system (Schreiber et al., 2006). Indeed, (Keller et al., 1996) observed systematic shifts in the movement fields of SC neurons when saccades were made to moving targets, concluding that an extrapolation pathway that may not include the SC may explain these observations.

\section{Comparison to literature}

Separate versus interacting systems

Until recently, saccades and pursuit have been separately characterized both functionally and anatomically. However, the past two decades of research have demonstrated that these two types of eye movements may originate from a single sensorimotor process (Krauzlis, 2004; Orban de Xivry and Lefèvre, 2007). Behavioral interactions between the saccadic and smooth pursuit system have been extensively investigated. The two systems are driven by both PE and RS inputs slip (Keller and Johnsen, 1990; de Brouwer et al., 2002b; Blohm et al., 2005; Etchells et al., 2010), supporting the use of both sensory estimates in the preparation of catch-up saccades. Moreover, both systems share a common predictive process mediated through the supplementary eye 
field (SEF; Nyffeler et al., 2008) and exhibit similar dependence on movement preparation time (Joiner and Shelhamer, 2006). The two systems are also intertwined anatomically and functionally. Frontal eye field (FEF) and SEF, LIP, and large brainstem and cerebellar pathways have both saccade and pursuit related activity (for review, see Orban de Xivry and Lefèvre, 2007). We further provide support for the hypothesis that the inputs to the saccadic and pursuit system are intertwined and used in combination to compute predictions of future PEs, and that these predictions are modulated by sensory uncertainty.

\section{Saccade triggering}

The LATER model accurately reproduces the trigger time distributions of saccades triggered to stationary targets observed in human participants (Noorani and Carpenter, 2016). However, the model does not account for the sensorimotor context such as target directions, pursuit gain, or RS. As discussed, saccades and pursuit are highly interacting systems. Krauzlis and Miles (1996) demonstrated that saccades and pursuit share the same inputs for the release of fixation from stationary targets on movement initiation. Perhaps the same is true for catch-up saccade triggering during smooth pursuit. Saccade trigger times to moving targets appear asymmetrical, as targets moving away from the fovea are triggered faster both during pursuit initiation and ongoing pursuit (de Brouwer et al., 2002a; Bieg et al., 2015). These trends are adequately captured by the accumulated confidence in predicted error model, while the presented behavioral results provide further evidence for a predictive and uncertainty dependent trigger mechanism as seen by saccade occurrence and trigger times. Thus, it appears that catch-up saccades are another outcome of a singular sensorimotor process that governs both smooth pursuit and saccadic eye movements.

\section{Decision making}

Visual and motor latencies account for $\sim 50 \%$ of the length and variability of saccade trigger times (Gold and Shadlen, 2007), suggesting a more complex decision process. Statistical decision making is an inference process whereby prior beliefs, incoming evidence, and time constraints are combined by a decision maker to choose between two or more competing hypotheses about the state of the world. Models of motor planning based on this theory use uncertain evidence and explicit action penalties and successfully predict human behavior (Trommershäuser et al., 2003). In the context of this study, the decision is to trigger or suppress a saccade. We hypothesized that confidence in future error estimates are used as a decision signal to coordinate the triggering of a saccade or to remain in smooth pursuit. signal detection theory (SDT), a common decision-making framework, incorporates a probability ratio of distributions representing parameters, observations, or competing hypotheses (Gold and Shadlen, 2007). The confidence in a particular decision is defined as the probability that a decision is correct given the evidence (Pouget et al., 2016). An extension of SDT is the sequential probability ratio test (SPRT), where sequential analysis of incoming samples accumulate over time to reach a positive or negative decision bound (Wald and Wolfowitz, 1948). Given a certain decision threshold, we expected to observe differences in trigger times based on the proposed decision mechanism. That is, a quicker rise to threshold given large or sustained predicted errors and high confidence in those estimates. Our results match our expectations since predicted error magnitudes before saccades inform whether a saccade will occur and what the trigger time will be. This is consistent with previous work that saccades and pursuit share a common decision signal but use different thresholds and are subject to different delays (Liston and Krauzlis, 2005). Thus, the motor control and coordination of saccades and smooth pursuit is viewed as a decision-making process.

\section{Implications for models}

When motor control in the brain is viewed as a decision-making process, it can be modeled using decision boundaries and evidence accumulation. Behavioral, neurophysiological, and computational research has revealed many links between decision-making processes and sensorimotor control (for review, see Wolpert and Landy, 2012; Gallivan et al., 2018). Models of decision making such as the LATER model have accurately predicted reaction time distributions for complex psychophysics tasks such as Go-NoGo, countermanding, and tasks where continuous evaluation of new sensory evidence is required (Noorani and Carpenter, 2016). In particular, the superior colliculus has been suggested to implement a decision process before the preparation of saccades (Ratcliff et al., 2003; Lo and Wang, 2006). Our model suggests a similar decision process of evidence accumulation takes place for the saccade trigger mechanism (Coutinho et al., 2018). The model predictions of saccade trigger times distributions are consistent with the behavioral results observed in this study. Furthermore, both our model and behavioral data provide new insights into how uncertainty in sensory inputs modulate oculomotor responses during tracking behavior. Our results imply that models must take into account information regarding prediction of future errors, crossing time information, and sensory noise to be able to account for all possible trigger behaviors.

\section{Motor control and uncertainty}

Neural control of movement is limited by noise from both sensory inputs such as vision and proprioception and from motor commands. However, the majority of motor variation in eye movements ( $92 \%)$ can be attributed to sensory noise such as errors of target motion or position estimates (Osborne et al., 2005). At the cognitive level we refer to this noise as uncertainty, which greatly influences our motor decisions. The central nervous system evolved to minimize the impact of sensory uncertainty by integrating information from numerous modalities and waiting to accumulate more information (van Beers et al., 2002). We proposed that uncertainty similarly modulates the catch-up saccade trigger mechanism, whereby highly uncertain stimuli such as a blurred or fast target will require further evidence accumulation and result in later 
or suppressed saccades. Similar perception-action tradeoffs in a ball-interception task under sensory uncertainty and time constraint are near Bayes-optimal (Faisal and Wolpert, 2009). Our model extends an existing Bayesian model of motion estimation and smooth pursuit using Kalman filtering (Orban de Xivry et al., 2013) with the addition of a future error estimation and a decision pathway for evidence accumulation. In the face of uncertainty, near-optimal Bayesian behavior has been previously observed in hand movement dynamics as well as perception (Stocker and Simoncelli, 2006; Acerbi et al., 2017) and a neural implementation of Bayesian estimation has been exhibited in the FEF during smooth pursuit (Darlington et al., 2018). Our model-guided behavioral results of how uncertainty modulates the saccade trigger decision are consistent with a Bayesian observer that optimally combines sensory inputs and their uncertainties to produce near optimal behavior. We further provide insight that the trigger mechanism likely relies on a probabilistic estimate of $\mathrm{PE}_{\text {pred }} \mathrm{s}$ through linear motion extrapolation.

\section{Underlying neurophysiology for prediction}

Motion extrapolation provides a useful mechanism for the brain to make sense of a continuously changing sensory environment. For example, catch-up saccades programming involves extrapolation of target position using current target velocity to land on the target by the time of saccade execution (de Brouwer et al., 2001; Lisi and Cavanagh, 2017). The FEFs were proposed to create a prediction map of stable visual percepts able to report and correct prediction errors following a saccade and were also correlated with saccadic trigger time (Connolly et al., 2005; Crapse and Sommer, 2008). Internal models of target motion are necessary to overcome visual delays inherent to the visuomotor system but also for instances of target occlusion and interceptive movements (Becker and Fuchs, 1985; Bosco et al., 2015). These internal models are useful as they allow the brain to minimize prediction error and behave optimally in the environment (McNamee and Wolpert, 2019). Notably, the eye position signals decoded from visual areas such as MT, MST, VIP, and LIP lead the current eye position, indicating a predictive process based on an efference copy of eye position (Dowiasch et al., 2016). This efference copy likely originates from area MST, where neurons account for effects of eye movements and can be used to inform saccade planning (Tanaka, 2005). Thus, it is highly plausible and supported by our results that prediction error plays a central role in the coordination of saccades during smooth pursuit. We observed that humans predict future errors by extrapolating $150 \mathrm{~ms}$ in the future, consistent with similar time estimates for smooth pursuit and memory guided saccades (Diaz et al., 2013; Orban de Xivry et al., 2013). Due to the heavily intertwined saccade and pursuit pathways, several brain areas are likely candidates to compute this prediction, such as the FEF/SEF or PPC. Other potential sites where extrapolation could occur by integrating position and velocity signals are the nucleus reticularis tegmenti pontis (NRTP) and the vermis cerebellum, as suggested by Schreiber et al. (2006). While previously suggested explanations for the saccade trigger such as $T_{x e}$ or $T_{x t}$ do not provide a simple mechanism, they remain behaviorally relevant. As shown, negative or positive crossing time indicates an increasing or decreasing $\mathrm{PE}_{\text {pred }}$, respectively. Although predicted error trigger times distributions are centered on zero, positive or negative $T_{x t}$ can widen or narrow the distribution.

\section{Conclusion}

In conclusion, we show that $\mathrm{PE}_{\text {pred }}$ and uncertainty in sensory estimates are used to make catch-up saccade trigger decisions, and that they adequately explain saccade trigger times and behavioral variability to abrupt changes of position and VSs. Our study complements a breadth of literature in support of uncertain motor decision-making and predictive behavior. to further investigate this decision mechanism, acceleration inputs should be considered as they are known to modulate the smooth pursuit response (Brostek et al., 2017). Effects of predicted errors and uncertainty on saccade programming of amplitude may also be apparent on closer examination. Additionally, adapting the double-step ramp task to more naturalistic tracking behavior may be necessary to reinforce the brain's use of this trigger mechanism. Models of saccade and pursuit should collapse previously independent pathways known to be shared by both systems and integrate a component of sensory uncertainty. Finally, a focus on the neural correlates of sensory prediction and/or confidence as well as where the trigger decision is made will serve to strengthen arguments for this proposed mechanism.

\section{References}

Acerbi L, Vijayakumar S, Wolpert DM (2017) Target uncertainty mediates sensorimotor error correction. PLoS One 12:e0170466.

Becker W, Fuchs AF (1985) Prediction in the oculomotor system: smooth pursuit during transient disappearance of a visual target. Exp Brain Res 57:562-575.

Bieg HJ, Chuang LL, Bülthoff HH, Bresciani J-P (2015) Asymmetric saccade reaction times to smooth pursuit. Exp Brain Res 233: 2527-2238.

Blohm G, Missal M, Lefèvre P (2005) Direct evidence for a position input to the smooth pursuit system. J Neurophysiol 94:712-721.

Bosco G, Delle Monache S, Gravano S, Indovina I, La Scaleia B, Maffei V, Zago M, Lacquaniti F (2015) Filling gaps in visual motion for target capture. Front Integr Neurosci 9:13.

Brainard DH (1997) The psychophysics toolbox. Spat Vis 10:433436.

Brostek L, Eggert T, Glasauer S (2017) Gain control in predictive smooth pursuit eye movements: evidence for an accelerationbased predictive mechanism. eNeuro 4:ENEURO.0343-16.2017.

Carpenter RHS, Williams MLL (1995) Neural computation of log likelihood in control of saccadic eye movements. Nature 377:5962.

Chang CJ, Jazayeri M (2018) Integration of speed and time for estimating time to contact. Proc Natl Acad Sci USA 115:E2879E2887.

Chen J, Valsecchi M, Gegenfurtner KR (2017) Attention is allocated closely ahead of the target during smooth pursuit eye movements: evidence from eeg frequency tagging. Neuropsychologia 102: 206-216.

Connolly JD, Goodale MA, Goltz HC, Munoz DP (2005) FMRI activation in the human frontal eye field is correlated with saccadic reaction time. J Neurophysiol 94:605-611. 
Coutinho JD, Lefèvre P, Blohm G (2018) Confidence in predicted position error explains saccadic decisions during pursuit. bioRxiv 396788.

Crapse TB, Sommer MA (2008) The frontal eye field as a prediction map. Prog Brain Res 171:383-390.

Darlington TR, Beck JM, Lisberger SG (2018) Neural implementation of Bayesian inference in a sensorimotor behavior. Nat Neurosci 21:1442-1451.

de Brouwer S, Missal M, Lefèvre P (2001) Role of retinal slip in the prediction of target motion during smooth and saccadic pursuit. $J$ Neurophysiol 86:550-558.

de Brouwer S, Missal M, Barnes G, Lefèvre P (2002a) Quantitative analysis of catch-up saccades during sustained pursuit. J Neurophysiol 87:1772-1780.

de Brouwer S, Yuksel D, Blohm G, Missal M, Lefèvre P (2002b) What triggers catch-up saccades during visual tracking? J Neurophysiol 87:1646-1650.

Diaz G, Cooper J, Rothkopf C, Hayhoe M (2013) Saccades to future ball location reveal memory-based prediction in a virtual-reality interception task. J Vis 13:20.

Dowiasch S, Blohm G, Bremmer F (2016) Neural correlate of spatial (mis-)localization during smooth eye movements. Eur J Neurosci 44:1846-1855.

Etchells PJ, Benton CP, Ludwig CJH, Gilchrist ID (2010) The target velocity integration function for saccades. J Vis 10:7.

Faisal AA, Wolpert DM (2009) Near optimal combination of sensory and motor uncertainty in time during a naturalistic perceptionaction task. J Neurophysiol 101:1901-1912.

Fooken J, Yeo S-H, Pai DK, Spering M (2016) Eye movement accuracy determines natural interception strategies. $J$ Vis 16:1.

Gallivan J, Chapman C, Wolpert D, Flanagan J (2018) Decisionmaking in sensorimotor control. Nat Rev Neurosci 19:519-534.

Goffart L, Cecala AL, Gandhi NJ (2017) The superior colliculus and the steering of saccades toward a moving visual target. J Neurophysiol 118:2890-2901.

Gold JI, Shadlen MN (2007) The neural basis of decision making. Annu Rev Neurosci 30:535-574.

Heinen SJ, Potapchuk E, Watamaniuk SNJ (2016) A foveal target increases catch-up saccade frequency during smooth pursuit. $J$ Neurophysiol 115:1220-1227.

Joiner WM, Shelhamer M (2006) Pursuit and saccadic tracking exhibit a similar dependence on movement preparation time. Exp Brain Res 173:572-586.

Keller E, Johnsen SDS (1990) Velocity prediction in corrective saccades during smooth-pursuit eye movements in monkey. Exp Brain Res 80:525-531.

Keller EL, Gandhi NJ, Weir PT (1996) Discharge of superior collicular neurons during saccades made to moving targets. J Neurophysiol 76:3573-3577.

Khan AZ, Lefèvre P, Heinen SJ, Blohm G (2010) The default allocation of attention is broadly ahead of smooth pursuit. J Vis 10:7.

Krauzlis RJ, Lisberger SG (1994a) A model of visually-guided smooth pursuit eye movements based on behavioral observations. J Comput Neurosci 1:265-283.

Krauzlis RJ, Lisberger SG (1994b) Temporal properties of visual motion signals for the initiation of smooth pursuit eye movements in monkeys. J Neurophysiol 72:150-162.

Krauzlis RJ, Miles FA (1996) Release of fixation for pursuit and saccades in humans: evidence for shared inputs acting on different neural substrates. J Neurophysiol 76:2822-2833.

Krauzlis RJ (2004) Recasting the smooth pursuit eye movement system. J Neurophysiol 91:591-603.

Lisi M, Cavanagh P (2017) Cooperative interactions between saccadic and pursuit planning when targeting a moving object. $\mathrm{J}$ Vis $17: 1278$.
Liston D, Krauzlis RJ (2005) Shared decision signal explains performance and timing of pursuit and saccadic eye movements. J Vis $5: 3$.

Lo CC, Wang XJ (2006) Cortico-basal ganglia circuit mechanism for a decision threshold in reaction time tasks. Nat Neurosci 9:956963.

McNamee D, Wolpert DM (2019) Internal models in biological control. Annu Rev Control Robot Auton Syst 2:339-364.

Melcher D, Kowler E (1999) Shapes, surfaces, and saccades. Vision Res 39:2929-2946.

Noorani I, Carpenter RHS (2016) The later model of reaction time and decision. Neurosci Biobehav Rev 64:229-251.

Nyffeler T, Rivaud-Pechoux S, Wattiez N, Gaymard B (2008) Involvement of the supplementary eye field in oculomotor predictive behavior. J Cogn Neurosci 20:1583-1594.

Orban de Xivry JJ, Lefèvre P (2007) Saccades and pursuit: two outcomes of a single sensorimotor process. J Physiol 584:11-23.

Orban de Xivry JJ, Coppe S, Blohm G, Lefèvre P (2013) Kalman filtering naturally accounts for visually guided and predictive smooth pursuit dynamics. J Neurosci 33:17301-17313.

Osborne LC, Lisberger SG, Bialek W (2005) A sensory source for motor variation. Nature 437:412-416.

Pouget A, Drugowitsch J, Kepecs A (2016) Confidence and certainty: distinct probabilistic quantities for different goals. Nat Neurosci 19:366-374.

Ratcliff R, Cherian A, Segraves M (2003) A comparison of macaque behavior and superior colliculus neuronal activity to predictions from models of two-choice decisions. J Neurophysiol 90:13921407.

Reddi BAJ, Carpenter RHS (2000) The influence of urgency on decision time. Nat Neurosci 3:827-830.

Robinson DA (1986) The systems approach to the oculomotor system. Vision Res 26:91-99.

Schiller PH (1986) The central visual system. Vision Res 26:13511386.

Schreiber C, Missal M, Lefèvre P (2006) Asynchrony between position and motion signals in the saccadic system. J Neurophysiol 95:960-969.

Schütz AC, Souto D (2011) Adaptation of catch-up saccades during the initiation of smooth pursuit eye movements. Exp Brain Res 209:537-549.

Shadmehr R, Smith MA, Krakauer JW (2010) Error correction, sensory prediction, and adaptation in motor control. Annu Rev Neurosci 33:89-108.

Stocker AA, Simoncelli EP (2006) Noise characteristics and prior expectations in human visual speed perception. Nat Neurosci 9:578-585

Tanaka M (2005) Involvement of the central thalamus in the control of smooth pursuit eye movements. J Neurosci 25:5866-5876.

Trommershäuser J, Maloney LT, Landy MS (2003) Statistical decision theory and trade-offs in the control of motor response. Spat Vis 16:255-275.

van Beers RJ, Baraduc P, Wolpert DM (2002) Role of uncertainty in sensorimotor control. Philos Trans R Soc London Ser B Biol Sci 357:1137-1145.

Wagenmakers EJ (2007) A practical solution to the pervasive problems of $p$ values. Psychonomic bulletin \& review 14:779-804.

Wald A, Wolfowitz J (1948) Optimum character of the sequential probability ratio test. Ann Math Stat 19:326-339.

Wolpert DM, Landy MS (2012) Motor control is decision-making. Curr Opin Neurobiol 22:996-1003.

Wurtz RH, Optican LM (1994) Superior colliculus cell types and models of saccade generation. Curr Opin Neurobiol 4:857-861. 